\title{
Advanced Synthesis of Dihydrofurans. Effect of Formic Acid on the Mn(III)-Based Oxidation
}

\author{
Risa Matsumoto and Hiroshi Nishino* \\ Department of Chemistry, Graduate School of Science and Technology, Kumamoto University \\ Kurokami 2-39-1, Chûou-Ku, Kumamoto 860-8555, Japan \\ Fax: +81-96-342-3374; E-mail: nishino@sci.kumamoto-u.ac.jp
}

\section{Supplementary Information}

Measurements, materials, IR, ${ }^{1} \mathrm{H}$ and ${ }^{13} \mathrm{C}$ NMR spectra, DEPT and HRMS data including copies of ${ }^{1} \mathrm{H}$ and ${ }^{13} \mathrm{C}$ NMR spectra for $5 \mathbf{b a}, 5 \mathbf{b b}, 5 \mathbf{b c}, 5 \mathbf{b d}, 5 \mathbf{d a}$, and 7-10.

* Received in

Address correspondence to H. Nishino, Department of Chemistry, Graduate School of Science and Technology, Kumamoto University, Kurokami 2-39-1, Chûou-Ku, Kumamoto 860-8555, Japan, Fax: +81-96-342-3374; E-mail:

nishino@sci.kumamoto-u.ac.jp 


\section{EXPERIMENTAL}

\section{Measurements}

Melting points were taken using a Yanagimoto micromelting point apparatus and are uncorrected. The NMR spectra were recorded using a JNM ECX 500 or AL300 FT-NMR spectrometer at 500 or $300 \mathrm{MHz}$ for ${ }^{1} \mathrm{H}$ and at 125 or $75 \mathrm{MHz}$ for ${ }^{13} \mathrm{C}$, with tetramethylsilane as the internal standard. The chemical shifts are reported as $\delta$ values (ppm) and the coupling constants in Hz. The following abbreviations are used for the multiplicities; s, singlet; d, doublet; t, triplet; q, quartet; m, multiplet; brs, broad singlet for the ${ }^{1} \mathrm{H}$ NMR spectrum. The IR spectra were measured in $\mathrm{CHCl}_{3}$ or $\mathrm{KBr}$ using a Shimadzu 8400 FT IR spectrometer and expressed in $\mathrm{cm}^{-1}$. The EI MS spectra were obtained by a Shimadzu QP-5050A gas chromatograph-mass spectrometer at the ionizing voltage of $70 \mathrm{eV}$. The high-resolution mass spectra and the elemental analyses were performed at the Instrumental Analysis Center, Kumamoto University, Kumamoto, Japan.

\section{Materials.}

Manganese(II) acetate tetrahydrate, $\mathrm{Mn}(\mathrm{OAc})_{2} \bullet 4 \mathrm{H}_{2} \mathrm{O}$, was purchased from Wako Pure Chemical Ind., Ltd. Manganese(III) acetate dihydrate, $\mathrm{Mn}(\mathrm{OAc})_{3} \cdot 2 \mathrm{H}_{2} \mathrm{O}$, was prepared according to our modified method. ${ }^{[12]}$ Nitrilotris(ethane-2,1-diyl) tris(3-oxobutanoate) (1) was prepared by the imidazole-catalyzed reaction of 2,2',2"-nitrilotriethanol with diketene in dry tetrahydrofuran at room temperature. The diarylethenes 2a-d were prepared by the Grignard reaction of the corresponding arylmagnesium bromides with acetophenones followed by acid-catalyzed dehydration. Imidazole, 2,2',2"-nitrilotriethanol, diketene, propyl 3-oxobutanoate (4c), and butyl 3-oxobutanoate (4e) were purchased from Tokyo Kasei Co., Ltd., and methyl 3-oxobutanoate (4a), ethyl 3-oxobutanoate (4b), isopropyl 3-oxobutanoate (4d), 2,4-pentanedione (6a), malonic acid (6b), and diethyl malonate (6c) were from Wako Pure Chemical Ind., Ltd., and used as received. Flash column chromatography was performed on silica gel 60N (40-50 $\mu \mathrm{m})$, which was purchased from Kanto Chemical Co., Inc., and preparative thin layer chromatography (TLC) on Wakogel B-10 (45 $\mu \mathrm{m})$ from Wako Pure Chemical Ind., Ltd. The solvents were commercially available first grade and used as received.

The physical data of the known products $\mathbf{5 b a}, \mathbf{5 b b}, \mathbf{5 b c}, \mathbf{5 b d}, \mathbf{5 d a}$, and 7-10 and references are listed below.

Ethyl 2-Methyl-5,5-diphenyl-4,5-dihydrofuran-3-carboxylate (5ba) ${ }^{[13]}$

Light yellow oil; $R_{\mathrm{f}}=0.4\left(\right.$ EtOAc-hexane, 2:8); IR $\left(\mathrm{CHCl}_{3}\right) v 1690(\mathrm{C}=\mathrm{O}) ;{ }^{1} \mathrm{H}$ NMR $\left(\mathrm{CDCl}_{3}\right) \delta 7.39-7.22(10 \mathrm{H}$, m, arom H), $4.15\left(2 \mathrm{H}, \mathrm{q}, J=7.2 \mathrm{~Hz}, \mathrm{CH}_{2}-\mathrm{CH}_{3}\right), 3.60\left(2 \mathrm{H}, \mathrm{s}, \mathrm{CH}_{2}\right), 2.35\left(3 \mathrm{H}, \mathrm{s}, \mathrm{CH}_{3}\right), 1.26(3 \mathrm{H}, \mathrm{t}, J=7.2 \mathrm{~Hz}, \mathrm{CH}-$ $\left.{ }_{2}-\underline{\mathrm{CH}}_{3}\right) ;{ }^{13} \mathrm{C} \mathrm{NMR}\left(\mathrm{CDCl}_{3}\right) \delta 166.2(\underline{\mathrm{C}}=\mathrm{O}), 165.8$ (=으), 145.1 (2C) (arom C), $128.3(4 \mathrm{C}), 127.4(2 \mathrm{C}), 125.6(4 \mathrm{C})$ (arom $\mathrm{CH}), 101.7(>\underline{\mathrm{C}}=), 91.4(>\underline{\mathrm{C}}<), 59.5\left(-\mathrm{OCH}_{2}\right), 44.1\left(-\underline{\mathrm{CH}}_{2}-\right), 14.4\left(\underline{\mathrm{CH}}_{3}\right), 14.2\left(\underline{\mathrm{CH}}_{3}\right) ;$ FAB HRMS (acetone/NBA) calcd for $\mathrm{C}_{20} \mathrm{H}_{21} \mathrm{O}_{3} 309.1491(\mathrm{M}+\mathrm{H})$. Found: 309.1510 .

\section{Ethyl 5,5-Bis(4-fluorophenyl)-2-methyl-4,5-dihydrofuran-3-carboxylate (5bb) ${ }^{[13]}$}

Colorless liquid; $R_{\mathrm{f}}=0.5$ (EtOAc-hexane, 1:9); IR $\left(\mathrm{CHCl}_{3}\right) v 1697(\mathrm{C}=\mathrm{O})$; ${ }^{1} \mathrm{H} \mathrm{NMR}\left(\mathrm{CDCl}_{3}\right) \delta 7.34-7.30(4 \mathrm{H}$, m, arom H), 7.04-6.99 (4H, m, arom H), $4.16\left(2 \mathrm{H}, \mathrm{q}, J=7.2 \mathrm{~Hz}, \underline{\mathrm{CH}}_{2}-\mathrm{CH}_{3}\right), 3.55\left(2 \mathrm{H}, \mathrm{q}, J=1.5 \mathrm{~Hz}, \mathrm{CH}_{2}\right), 2.34(3 \mathrm{H}, \mathrm{t}$, $\left.J=1.5 \mathrm{~Hz}, \underline{\mathrm{CH}}_{3}\right), 1.27\left(3 \mathrm{H}, \mathrm{t}, J=7.2 \mathrm{~Hz}, \mathrm{CH}_{2}-\underline{\mathrm{H}}_{3}\right) ;{ }^{13} \mathrm{C} \mathrm{NMR}\left(\mathrm{CDCl}_{3}\right) \delta 166.0(\underline{\mathrm{C}}=\mathrm{O}), 165.6(=\underline{\mathrm{CO}}-), 162.1(2 \mathrm{C})$ (arom $\underline{\mathrm{C}-\mathrm{F},} J=245.6 \mathrm{~Hz}) 140.8(2 \mathrm{C}, J=2.4 \mathrm{~Hz}$, arom C-1'), 127.5 (4C, $J=7.1 \mathrm{~Hz}$, arom C-2'), $115.2(4 \mathrm{C}, J=21.4 \mathrm{~Hz}$, arom C-3'), $101.8(>\underline{\mathrm{C}}=), 90.6(>\underline{\mathrm{C}}<), 59.7\left(-\mathrm{OCH}_{2}\right), 44.3\left(-\underline{\mathrm{CH}}_{2^{-}}\right), 14.4\left(\underline{\mathrm{CH}}_{3}\right), 14.2\left(\underline{\mathrm{CH}}_{3}\right)$.

Ethyl 5,5-Bis(4-chlorophenyl)-2-methyl-4,5-dihydrofuran-3-carboxylate (5bc) ${ }^{[14]}$

Colorless amorphous; $R_{\mathrm{f}}=0.5\left(\right.$ EtOAc-hexane, 1:9); IR $\left(\mathrm{CHCl}_{3}\right) v 1701(\mathrm{C}=\mathrm{O})$; ${ }^{1} \mathrm{H}$ NMR $\left(\mathrm{CDCl}_{3}\right) \delta 7.32-7.26$ $(8 \mathrm{H}, \mathrm{m}, \operatorname{arom~H}), 4.16\left(2 \mathrm{H}, \mathrm{q}, J=7.2 \mathrm{~Hz}, \mathrm{CH}_{2}-\mathrm{CH}_{3}\right), 3.53\left(2 \mathrm{H}\right.$, br. q, $\left.J=1.8 \mathrm{~Hz}, \mathrm{CH}_{2}\right), 2.33\left(3 \mathrm{H}, \mathrm{t}, J=1.8 \mathrm{~Hz}, \mathrm{CH}_{3}\right)$, 
$1.27\left(3 \mathrm{H}, \mathrm{t}, J=7.2 \mathrm{~Hz}, \mathrm{CH}_{2}-\mathrm{CH}_{3}\right) ;{ }^{13} \mathrm{C} \mathrm{NMR}\left(\mathrm{CDCl}_{3}\right) \delta 165.9(\underline{\mathrm{C}}=\mathrm{O}), 165.5$ (=으), $143.2(2 \mathrm{C})(\operatorname{arom~C}), 133.7(2 \mathrm{C})$ (arom C), $128.6(4 \mathrm{C}), 127.0(4 \mathrm{C})(\operatorname{arom~CH}), 101.8(>\underline{\mathrm{C}}=), 90.5(>\underline{\mathrm{C}}<), 59.7\left(-\mathrm{OCH}_{2^{-}}\right), 44.0\left(-\underline{\mathrm{CH}}_{2}-\right), 14.4\left(\underline{\mathrm{CH}}_{3}\right), 14.2$ $\left(\mathrm{CH}_{3}\right)$; FAB HRMS (acetone/NBA) calcd for $\mathrm{C}_{20} \mathrm{H}_{19} \mathrm{Cl}_{2} \mathrm{O}_{3} 377.0711(\mathrm{M}+\mathrm{H})$; found: 377.0692 .

Ethyl 5,5-Bis(4-methylphenyl)-2-methyl-4,5-dihydrofuran-3-carboxylate (5bd) ${ }^{[13]}$

Light yellow oil; $R_{\mathrm{f}}=0.5\left(\right.$ EtOAc-hexane, 1:9); IR $\left(\mathrm{CHCl}_{3}\right) v 1690(\mathrm{C}=\mathrm{O})$; ${ }^{1} \mathrm{H}$ NMR $\left(\mathrm{CDCl}_{3}\right) \delta 7.26-7.25(4 \mathrm{H}$, m, arom H), 7.13-7.12 (4H, m, arom H), $4.15\left(2 \mathrm{H}, \mathrm{q}, J=7.2 \mathrm{~Hz}, \underline{\mathrm{CH}}_{2}-\mathrm{CH}_{3}\right), 3.60\left(2 \mathrm{H}, \mathrm{q}, J=1.5 \mathrm{~Hz}, \mathrm{CH}_{2}\right), 2.33(3 \mathrm{H}, \mathrm{t}$, $\left.J=1.5 \mathrm{~Hz}, \underline{\mathrm{CH}}_{3}\right), 2.32\left(6 \mathrm{H}, \mathrm{s}, \mathrm{CH}_{3} \times 2\right), 1.26\left(3 \mathrm{H}, \mathrm{t}, J=7.2 \mathrm{~Hz}, \mathrm{CH}_{2}-\mathrm{CH}_{3}\right) ;{ }^{13} \mathrm{C} \mathrm{NMR}\left(\mathrm{CDCl}_{3}\right) \delta 166.3(\underline{\mathrm{C}}=\mathrm{O}), 165.9$ (=으), $142.4(2 \mathrm{C})(\operatorname{arom} \mathrm{C}), 137.1(2 \mathrm{C})\left(\operatorname{arom} \underline{\mathrm{CCH}}_{3}\right), 128.9(4 \mathrm{C}), 125.6(4 \mathrm{C})(\operatorname{arom} \mathrm{CH}), 101.7(>\underline{\mathrm{C}}=), 91.4(>\underline{\mathrm{C}}<)$, $59.5\left(-\mathrm{OCH}_{2}\right), 44.1\left(-\underline{\mathrm{CH}}_{2}-\right), 21.0,14.4,14.2\left(\underline{\mathrm{CH}}_{3}\right)$.

i-Propyl 2-Methyl-5,5-diphenyl-4,5-dihydrofuran-3-carboxylate $(5 \mathrm{da})^{[3 \mathrm{e}]}$

Light yellow oil; $R_{\mathrm{f}}=0.5($ EtOAc-hexane, $1: 9)$; IR $\left(\mathrm{CHCl}_{3}\right) v 1684(\mathrm{C}=\mathrm{O})$; ${ }^{1} \mathrm{H}$ NMR $\left(\mathrm{CDCl}_{3}\right) \delta 7.40-7.24(10 \mathrm{H}$, m, arom H), $5.04(1 \mathrm{H}, \mathrm{sep}, J=6.2 \mathrm{~Hz},>\mathrm{C} \underline{\mathrm{H}}-), 3.59\left(2 \mathrm{H}, \mathrm{q}, J=1.5 \mathrm{~Hz}, \mathrm{C}_{2}\right), 2.34\left(3 \mathrm{H}, \mathrm{t}, J=1.5 \mathrm{~Hz}, \mathrm{CH}_{3}\right), 1.24(6 \mathrm{H}, \mathrm{d}$, $\left.J=6.2 \mathrm{~Hz},-\mathrm{CH}\left(\mathrm{CH}_{3}\right)_{2}\right) ;{ }^{13} \mathrm{C} \mathrm{NMR}\left(\mathrm{CDCl}_{3}\right) \delta 165.9(\underline{\mathrm{C}}=\mathrm{O}), 165.4$ (=으-), 145.3 (2C) (arom C), $128.3(4 \mathrm{C}), 127.4(2 \mathrm{C})$, 125.7 (4C) (arom CH), $102.0(>\underline{\mathrm{C}}=), 91.3(>\underline{\mathrm{C}}<), 66.7(-\mathrm{OC} \underline{\mathrm{H}}<), 44.2\left(-\underline{\mathrm{CH}}_{2^{-}}\right), 22.1\left(-\mathrm{CH}\left(\underline{\mathrm{CH}}_{3}\right)_{2}\right), 14.2\left(\underline{\mathrm{CH}}_{3}\right) ; \mathrm{FAB}$ HRMS (acetone/NBA) calcd for $\mathrm{C}_{21} \mathrm{H}_{23} \mathrm{O}_{3} 323.1647(\mathrm{M}+\mathrm{H})$; found 323.1665 .

\section{3-Acetyl-2-methyl-5,5-diphenyl-4,5-dihydrofuran (7) ${ }^{[3 \mathrm{a}]}$}

Light yellow oil; $R_{\mathrm{f}}=0.3\left(\right.$ EtOAc-hexane, 1:9); IR $\left(\mathrm{CHCl}_{3}\right) v 1667(\mathrm{C}=\mathrm{O}) ;{ }^{1} \mathrm{H}$ NMR $\left(\mathrm{CDCl}_{3}\right) \delta 7.39-7.25(10 \mathrm{H}$,

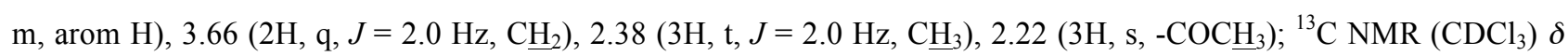
$194.1(\underline{\mathrm{C}}=\mathrm{O}), 165.9$ (=ㅁ-), 144.9 (2C) (arom C), 128.3 (4C), $127.6(2 \mathrm{C}), 125.6(4 \mathrm{C})(\operatorname{arom} \mathrm{CH}), 112.2(>\underline{\mathrm{C}}=), 91.5$ $(>\underline{\mathrm{C}}<), 44.8\left(-\underline{\mathrm{CH}}_{2^{-}}\right), 29.4\left(-\mathrm{COCH}_{3}\right), 15.2\left(\underline{\mathrm{CH}}_{3}\right)$.

\section{3,3,8,8-Tetraphenyl-2,7-dioxaspiro[4.4]nonane-1,6-dione $(8)^{[15]}$}

Colorless microcrystals (from $\mathrm{CHCl}_{3} /$ hexane); mp 287-290 ${ }^{\circ} \mathrm{C}$ (lit, mp 284.5-285.0 ${ }^{\circ} \mathrm{C}$ ); $R_{\mathrm{f}}=0.8\left(\mathrm{CHCl}_{3}\right) ; \mathrm{IR}$ $\left(\mathrm{CHCl}_{3}\right) v 1798,1667(\mathrm{C}=\mathrm{O})$; ' ${ }^{1} \mathrm{H}$ NMR $\left(\mathrm{CDCl}_{3}\right) \delta$ 7.43-7.35 $(6 \mathrm{H}, \mathrm{m}$, arom $\mathrm{H}), 7.31-7.23(14 \mathrm{H}, \mathrm{m}$, arom $\mathrm{H}), 3.01(2 \mathrm{H}$, $\left.\mathrm{d}, J=13.5 \mathrm{~Hz},-\mathrm{CH}_{2^{-}}\right), 2.61\left(2 \mathrm{H}, \mathrm{d}, J=13.5 \mathrm{~Hz},-\mathrm{CH}_{2^{-}}\right) ;{ }^{13} \mathrm{C} \mathrm{NMR}\left(\mathrm{CDCl}_{3}\right) \delta 173.5(\underline{\mathrm{C}}=\mathrm{O} \times 2), 142.1(2 \mathrm{C}), 142.0(2 \mathrm{C})$ (arom C), 128.9 (4C), $128.6(4 \mathrm{C}), 128.5$ (2C), 128.3 (2C), 125.5 (4C), 125.4 (4C) (arom CH), $88.60\left(\mathrm{Ph}_{2} \mathrm{CO}-\times 2\right), 53.9$ $(>\underline{\mathrm{C}}<), 45.6\left(-\mathrm{CH}_{2}-\times 2\right)$.

\section{Ethyl 2-oxo-5,5-diphenyl-2,5-dihydrofuran-3-carboxylate $(9)^{[8 \mathrm{e}, 9]}$}

Colorless microcrystals (from EtOH); mp 105-108 ${ }^{\circ} \mathrm{C}$ (lit, mp 107.9-109.4 $\left.{ }^{\circ} \mathrm{C}\right) ; R_{\mathrm{f}}=0.2($ EtOAc-hexane, 1:9); IR $\left(\mathrm{CHCl}_{3}\right) v 1780(\mathrm{C}=\mathrm{O}), 1719(\mathrm{C}=\mathrm{O}), 1267(\mathrm{C}-\mathrm{O}-\mathrm{C}) ;{ }^{1} \mathrm{H}$ NMR $\left(\mathrm{CDCl}_{3}\right) \delta 8.60(1 \mathrm{H}, \mathrm{s},>\mathrm{CH}-), 7.41-7.26(10 \mathrm{H}, \mathrm{m}$, arom $\mathrm{H}), 4.36\left(2 \mathrm{H}, \mathrm{q}, J=7.0 \mathrm{~Hz}, \underline{\mathrm{CH}}_{2}\right), 1.37\left(3 \mathrm{H}, \mathrm{t}, J=7.0 \mathrm{~Hz},-\mathrm{CH}_{2} \underline{\mathrm{CH}}_{3}\right) ;{ }^{13} \mathrm{C} \mathrm{NMR}\left(\mathrm{CDCl}_{3}\right) \delta 166.5(\underline{\mathrm{C}}=\mathrm{O}), 164.5(=\mathrm{C}-)$, $160.1(\mathrm{C}=\mathrm{O}), 138.1(2 \mathrm{C})(\operatorname{arom} \mathrm{C}), 129.0(4 \mathrm{C}), 128.9(2 \mathrm{C}), 126.6(4 \mathrm{C})(\operatorname{arom~CH}), 123.7(>\underline{\mathrm{C}}=), 89.2(>\underline{\mathrm{CPh}}), 61.89$ $\left(-\mathrm{CH}_{2} \mathrm{CH}_{3}\right), 14.1\left(-\mathrm{CH}_{2} \underline{\mathrm{CH}_{3}}\right)$.

\section{Diethyl 2-(2,2-diphenylethenyl)malonate $(10)^{[9,16]}$}

Colorless liquid; $R_{\mathrm{f}}=0.3$ (EtOAc-hexane, 1:9); IR $\left(\mathrm{CHCl}_{3}\right) v 1734(\mathrm{C}=\mathrm{O})$; ${ }^{1} \mathrm{H}$ NMR $\left(\mathrm{CDCl}_{3}\right) \delta 7.41-7.34(3 \mathrm{H}$, m, arom H), $7.29\left(5 \mathrm{H}, \mathrm{s}\right.$, arom H), 7.23-7.201 $\left(2 \mathrm{H}, \mathrm{m}\right.$, arom H), $6.33\left(1 \mathrm{H}, \mathrm{d}, J=11.0 \mathrm{~Hz},-\mathrm{CH}=\mathrm{Ph}_{2}\right), 4.21(4 \mathrm{H}, \mathrm{q}, J=$ $\left.7.0 \mathrm{~Hz},-\mathrm{CH}_{2} \mathrm{CH}_{3} \times 2\right), 4.19\left(1 \mathrm{H}, \mathrm{d}, J=11.0 \mathrm{~Hz},>\mathrm{C} \underline{\mathrm{H}}-\mathrm{CO}_{2} \mathrm{Et}\right), 1.27\left(6 \mathrm{H}, \mathrm{t}, J=7.0 \mathrm{~Hz},-\mathrm{CHCH}_{3} \times 2\right) ;{ }^{13} \mathrm{C} \mathrm{NMR}\left(\mathrm{CDCl}_{3}\right)$ $\delta 168.3(\underline{\mathrm{C}}=\mathrm{O} \times 2), 141.3(2 \mathrm{C})(\operatorname{arom} \mathrm{C}), 138.5\left(-\mathrm{CH}=\underline{\mathrm{CPh}}_{2}\right), 129.8(2 \mathrm{C}), 128.4(2 \mathrm{C}), 128.1(2 \mathrm{C}), 127.8(2 \mathrm{C}), 127.6$ (2C) $(\operatorname{arom~CH}), 119.7\left(-\underline{\mathrm{CH}}=\mathrm{CPh}_{2}\right), 61.5\left(-\underline{\mathrm{CH}}_{2} \mathrm{CH}_{3} \times 2\right), 53.0(>\underline{\mathrm{CH}}-), 14.0\left(-\mathrm{CH}_{2} \underline{\mathrm{CH}} \mathrm{H}_{3} \times 2\right)$. 


\section{REFERENCES}

13. Yamada, T.; Iwahara, Y.; Nishino, H.; Kurosawa, K. J. Chem. Soc., Perkin Tran. 1, 1993, 609-616.

14. Tategami, S.; Yamada, T.; Nishino, H.; Korp, J. D.; Kurosawa, K. Tetrahedron Lett. 1990, 31, 6371-6374.

15. Ito, N.; Nishino, H.; Kurosawa, K. Bull. Chem. Soc. Jpn. 1983, 56, 3527-3528.

16. Inés, B.; Palomas, D.; Holle, S.; Steinberg, S.; Nicasio, J. A.; Alcarazo, M. Angew. Chem. Int. Ed. 2012, 51, 12367-12369. 


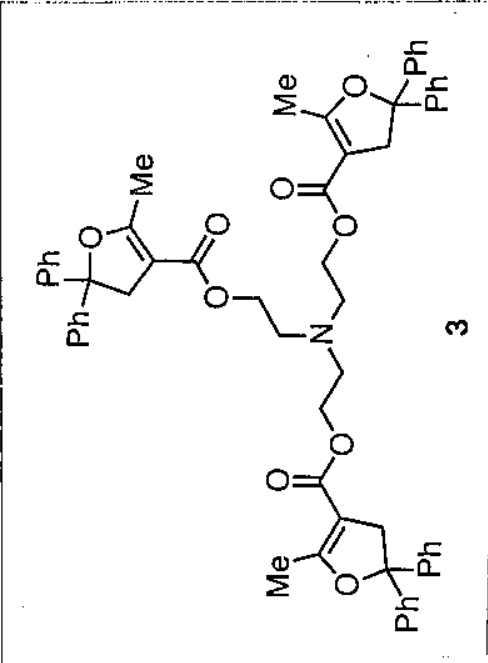

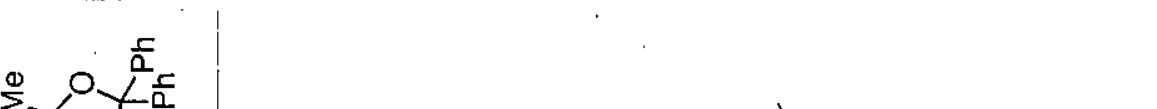

$00^{\circ} \mathcal{E}$

$8 \pm$

$\rightarrow$

$0^{\circ} \mathcal{E}$

藮

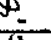

EE: 7

$\stackrel{\infty}{\infty}$

$\varepsilon 0 \varepsilon " z$

品

点

茲

' 1

ज্

$00: z$

$9.6^{\circ} \mathrm{L}$

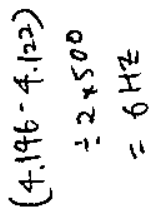




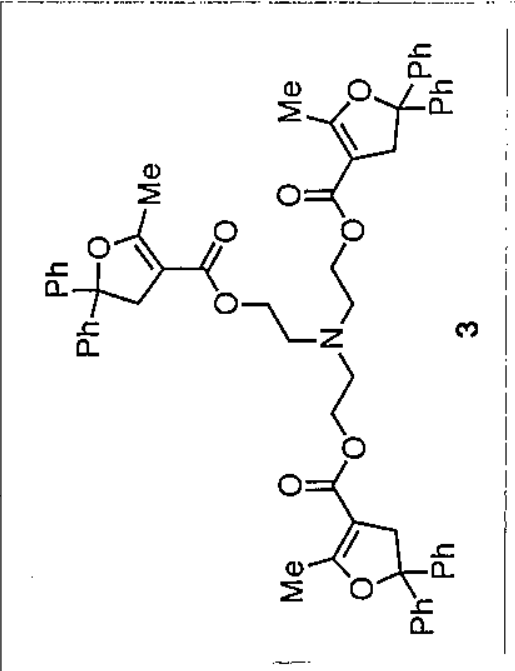




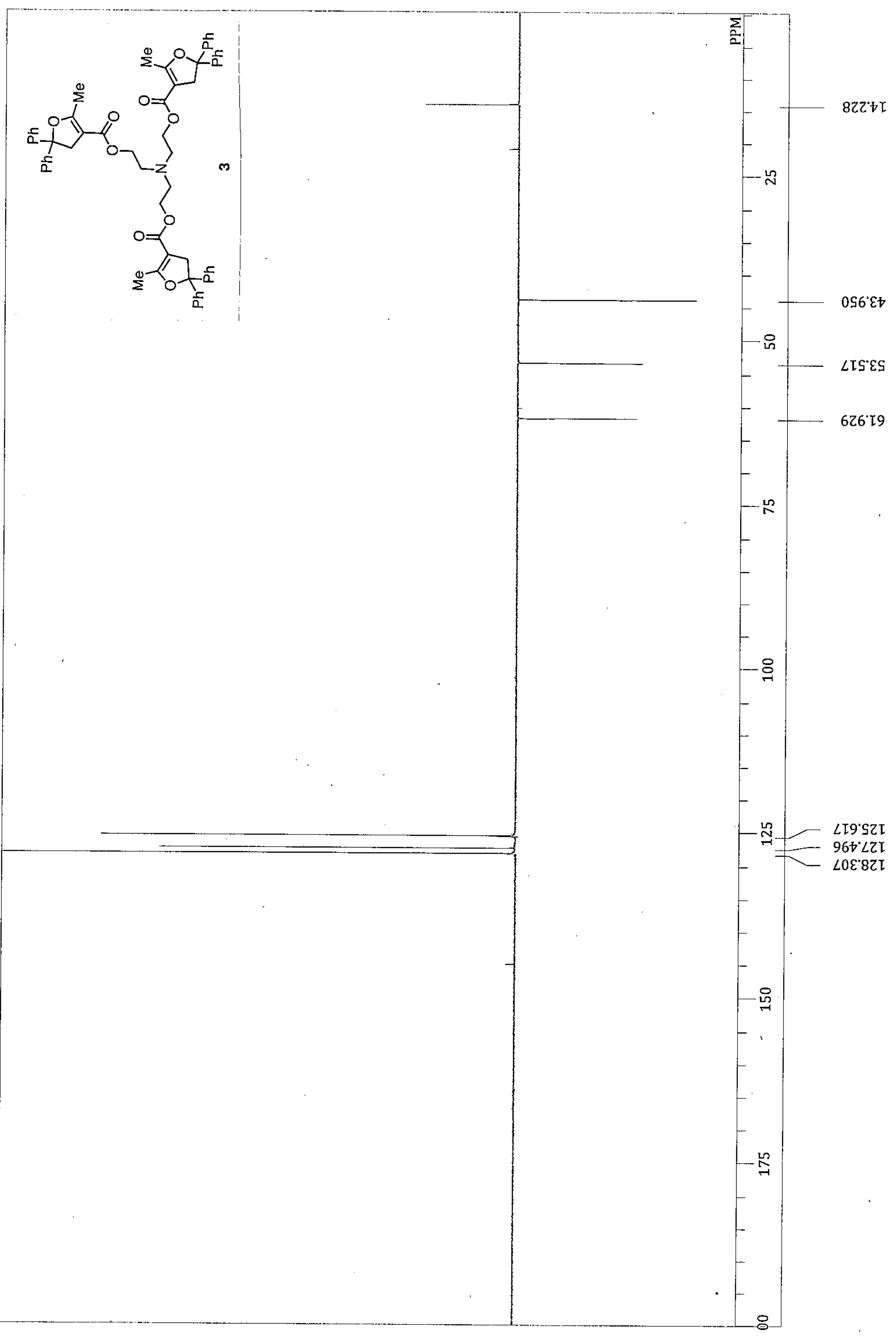



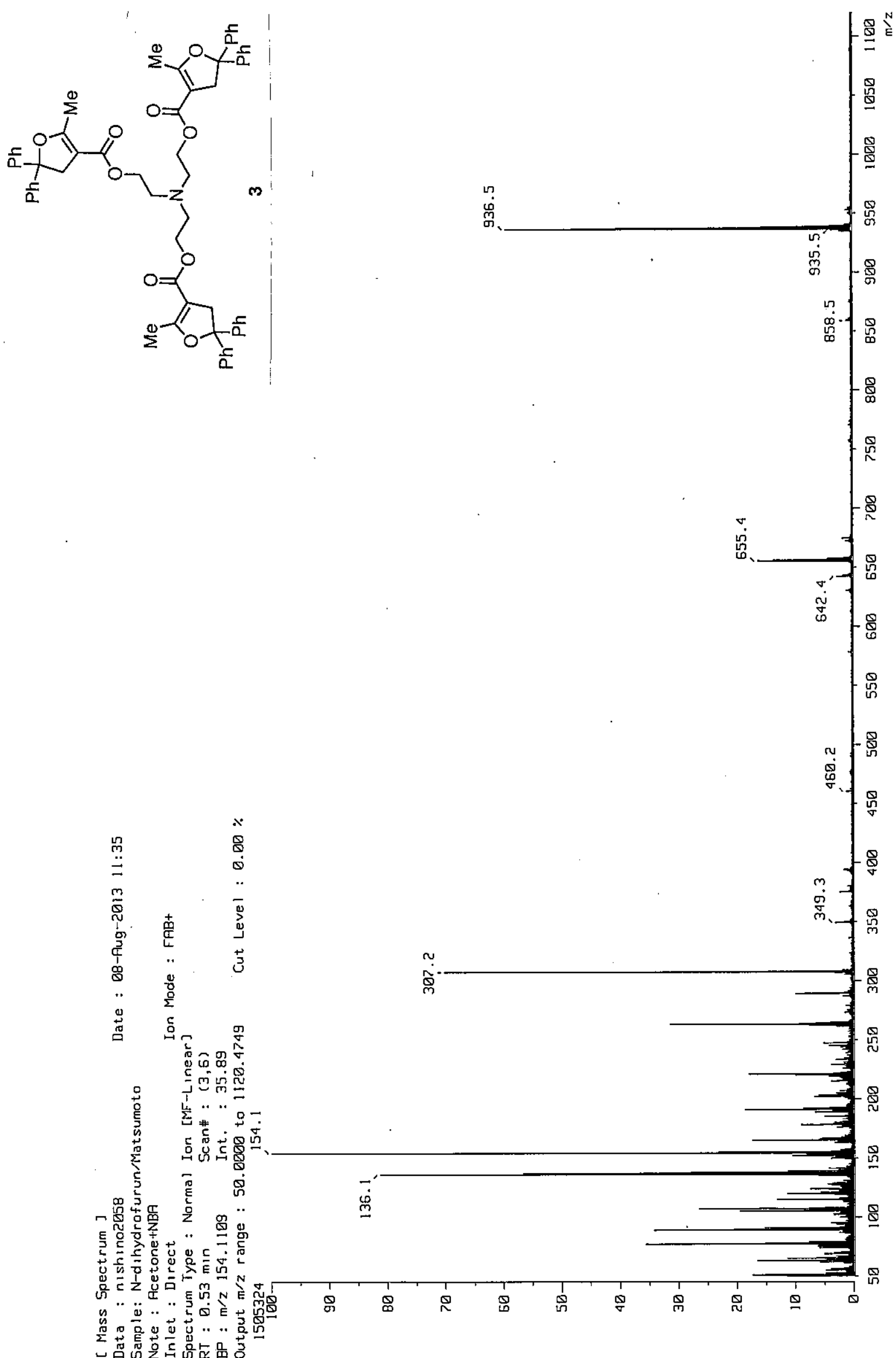


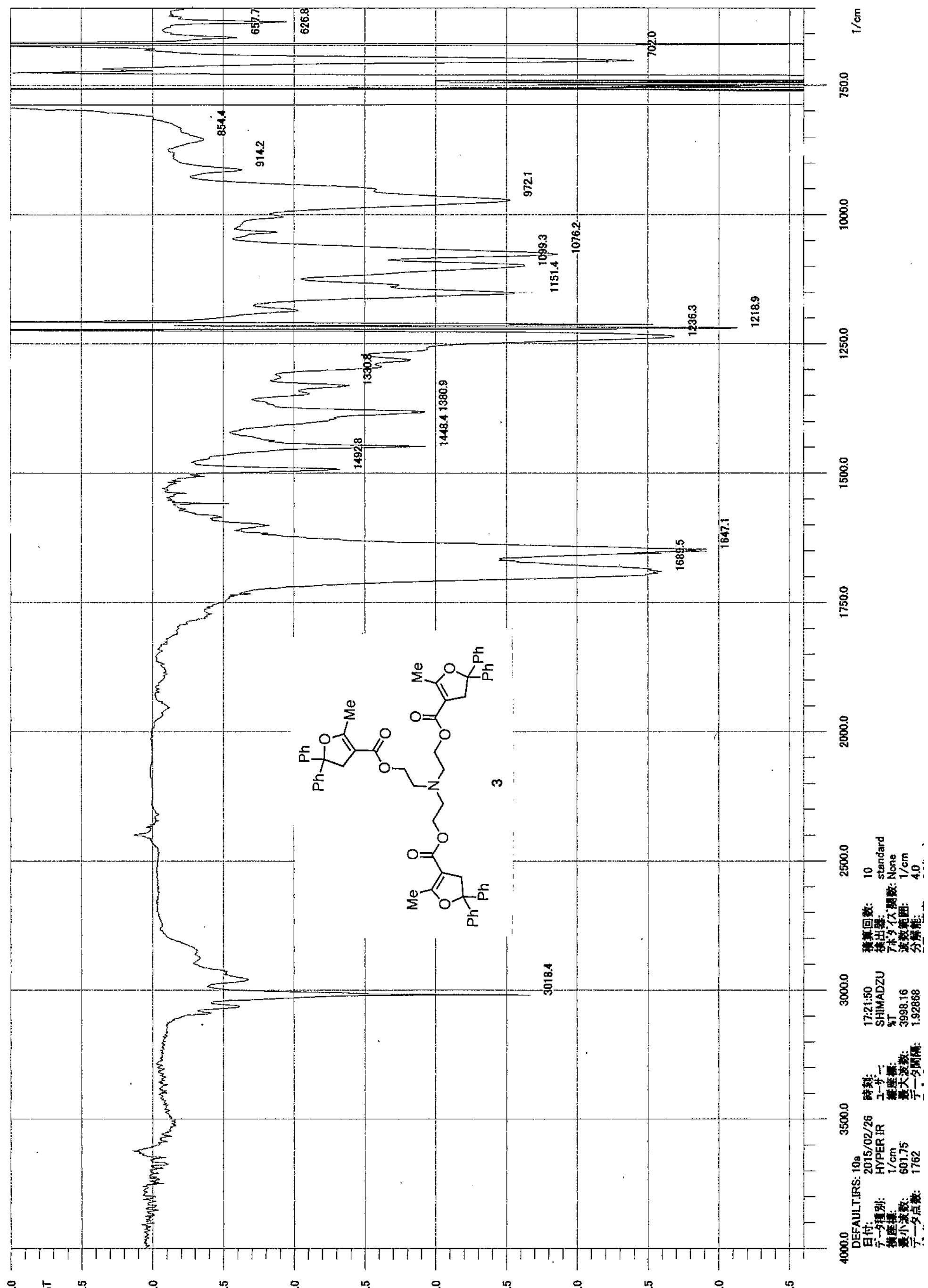

字 

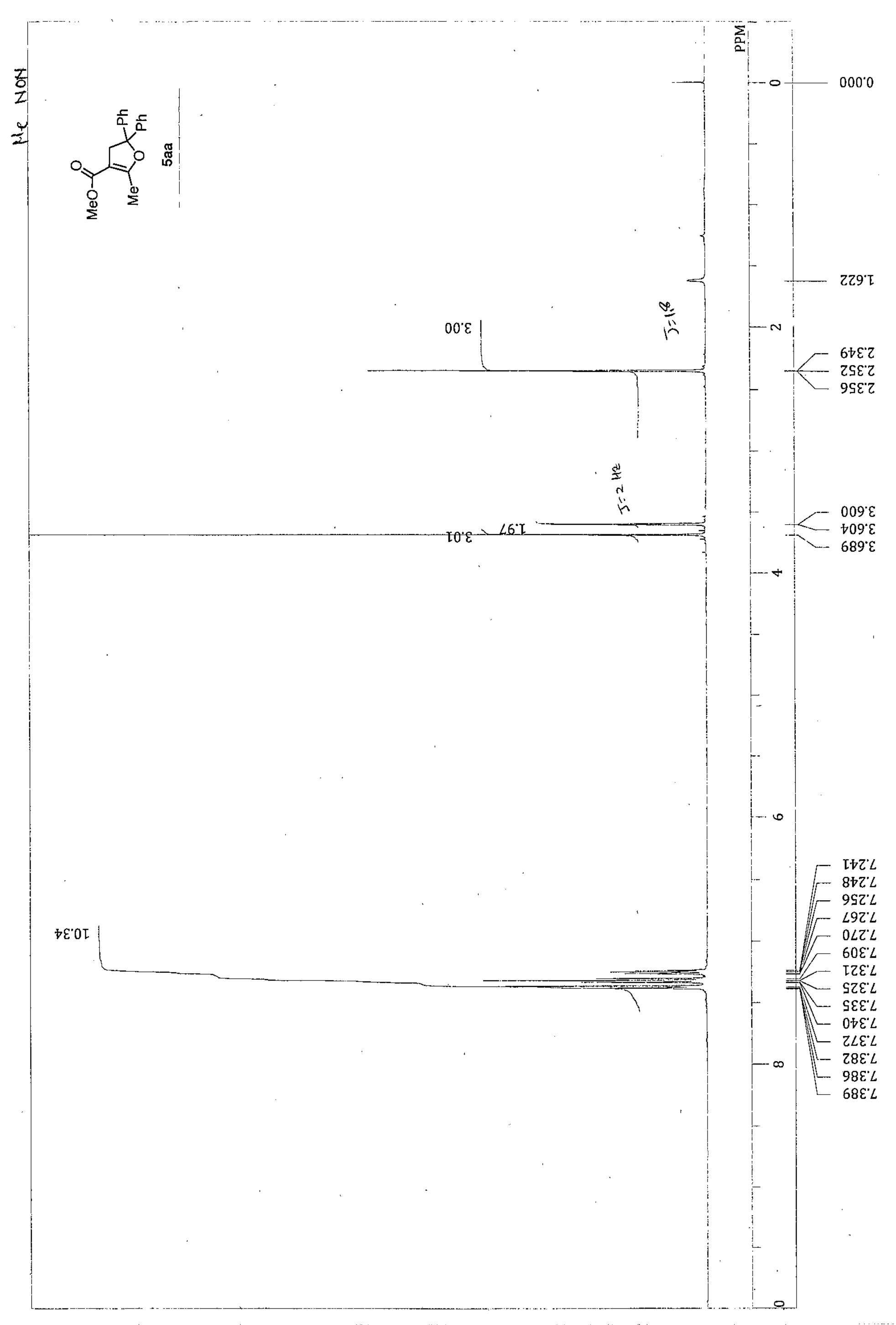
<smiles>COC(=O)C1=C(C)C(C)(C(F)(F)F)OC1C</smiles> 
$\frac{5}{11}$
$\frac{11}{2}$
$\frac{2}{2}$<smiles>COC(=O)C1=C(O)CC(C)(C)C1</smiles>

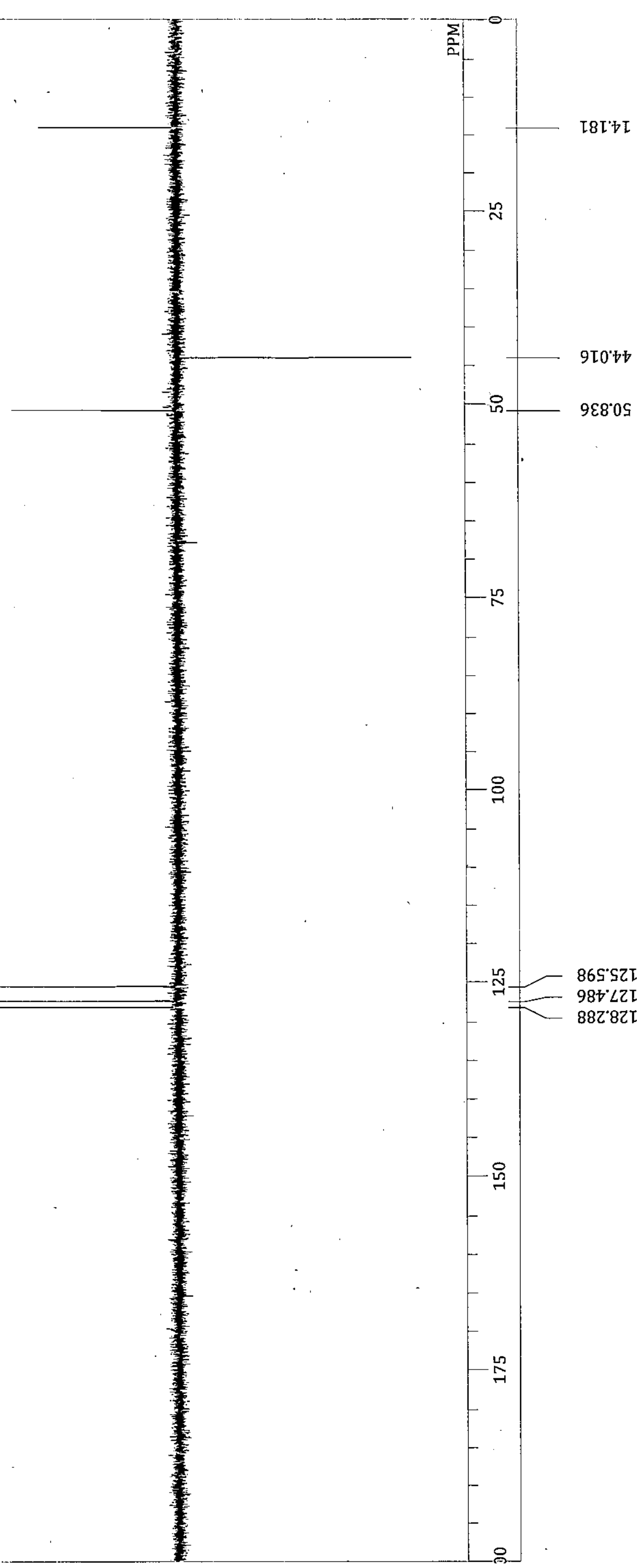




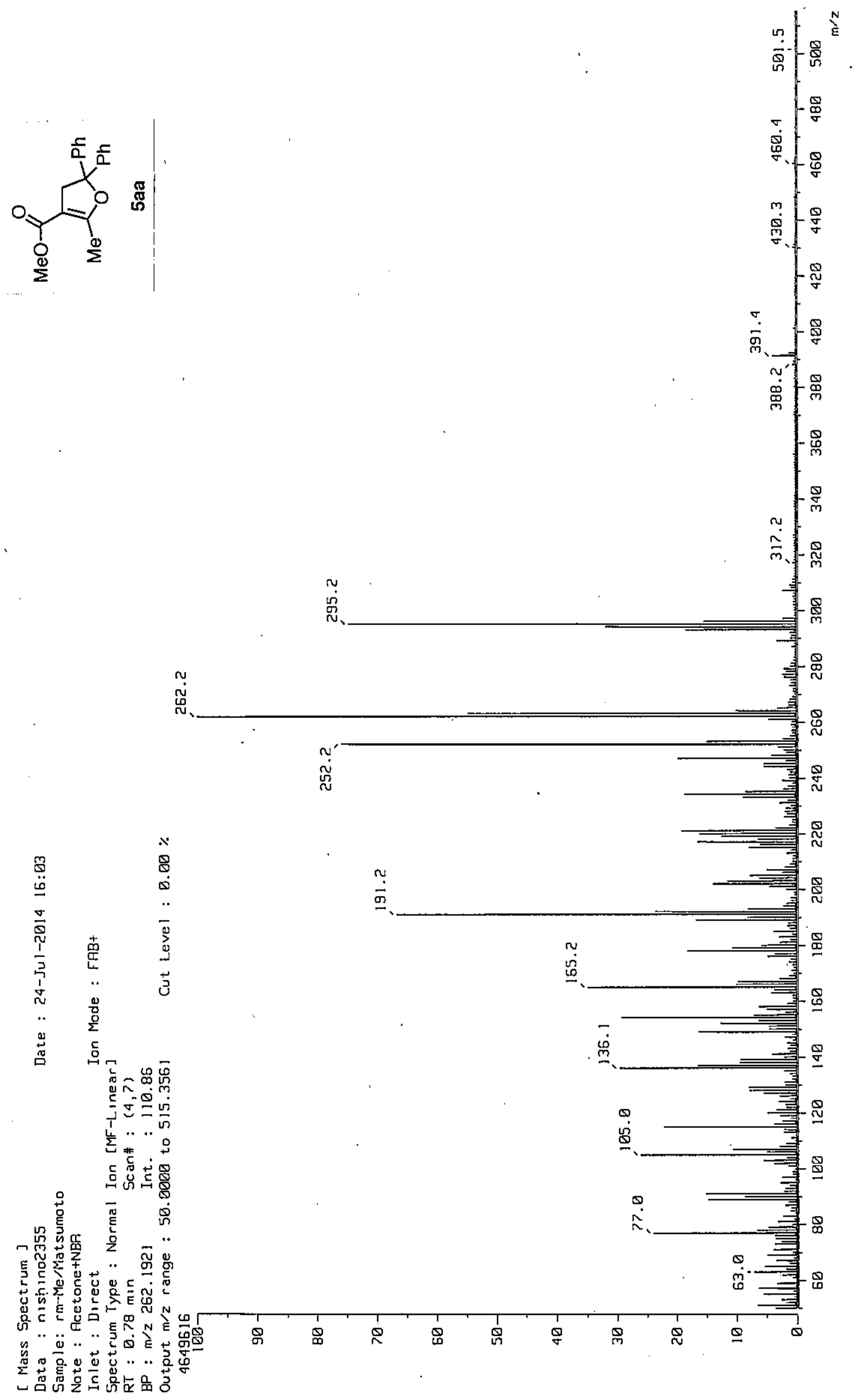




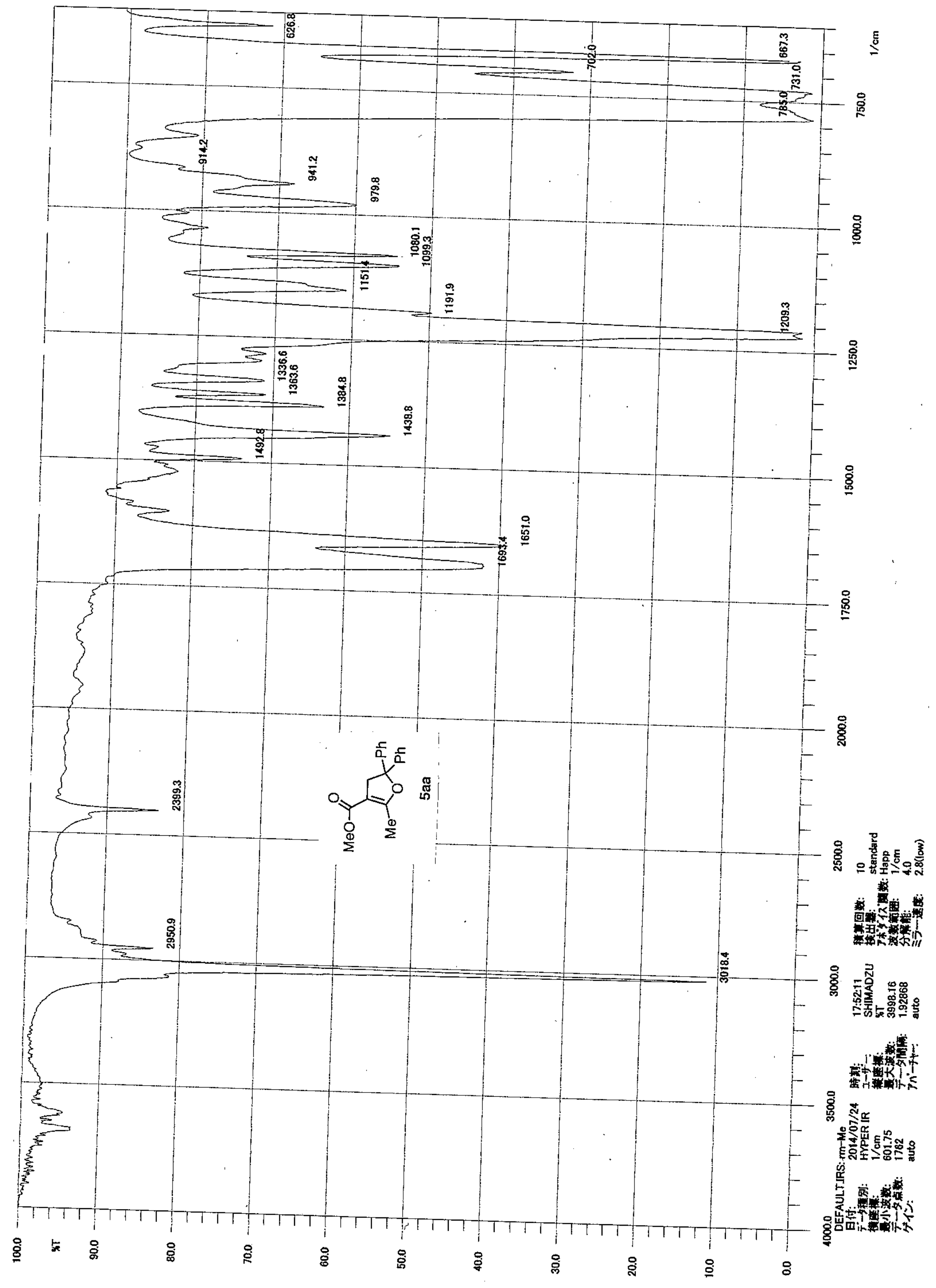


(2)

002

86:

85'0 

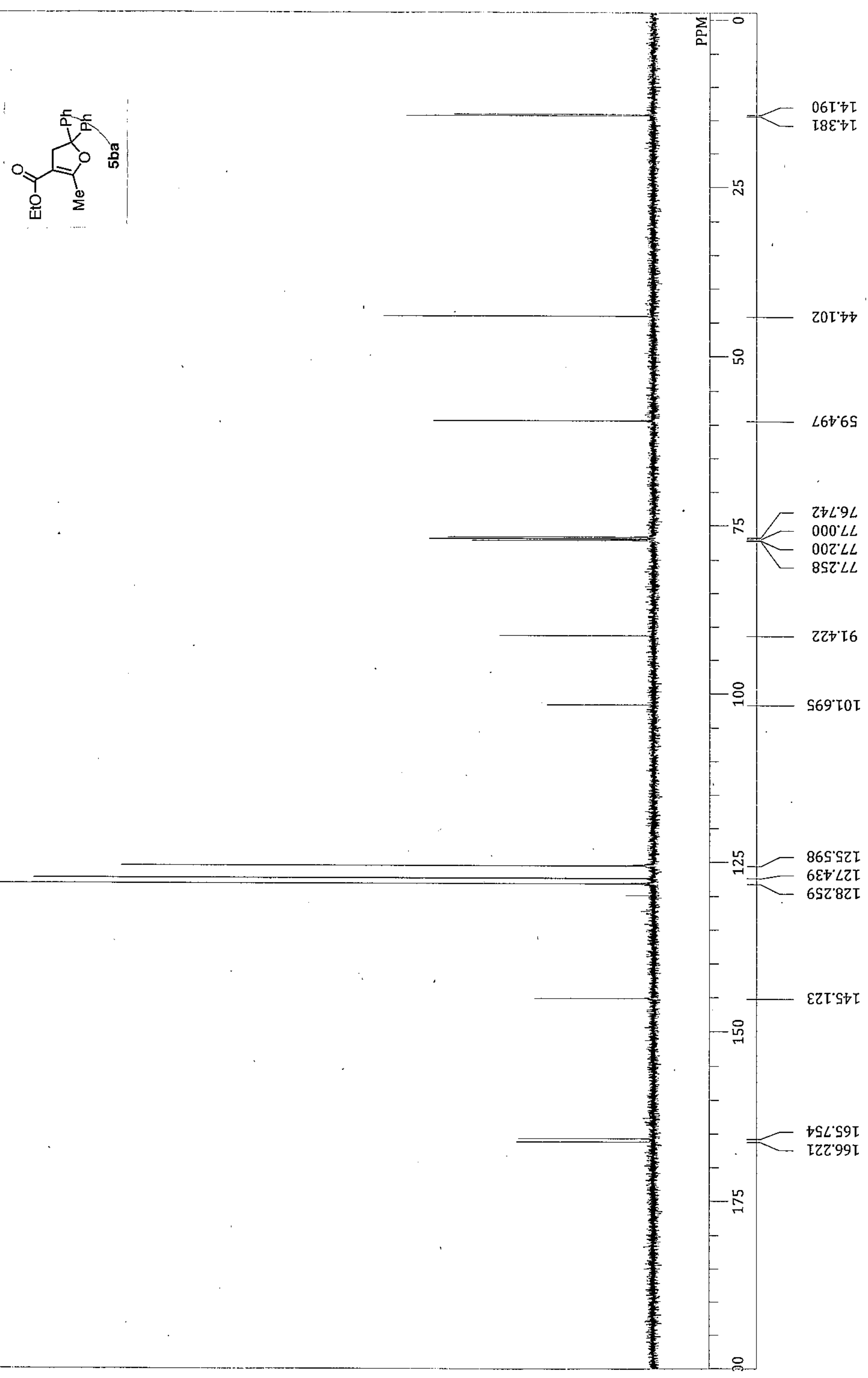
5
5
步
0
古

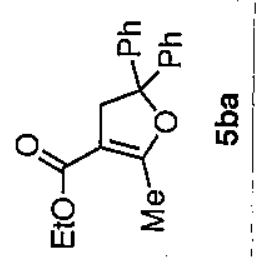



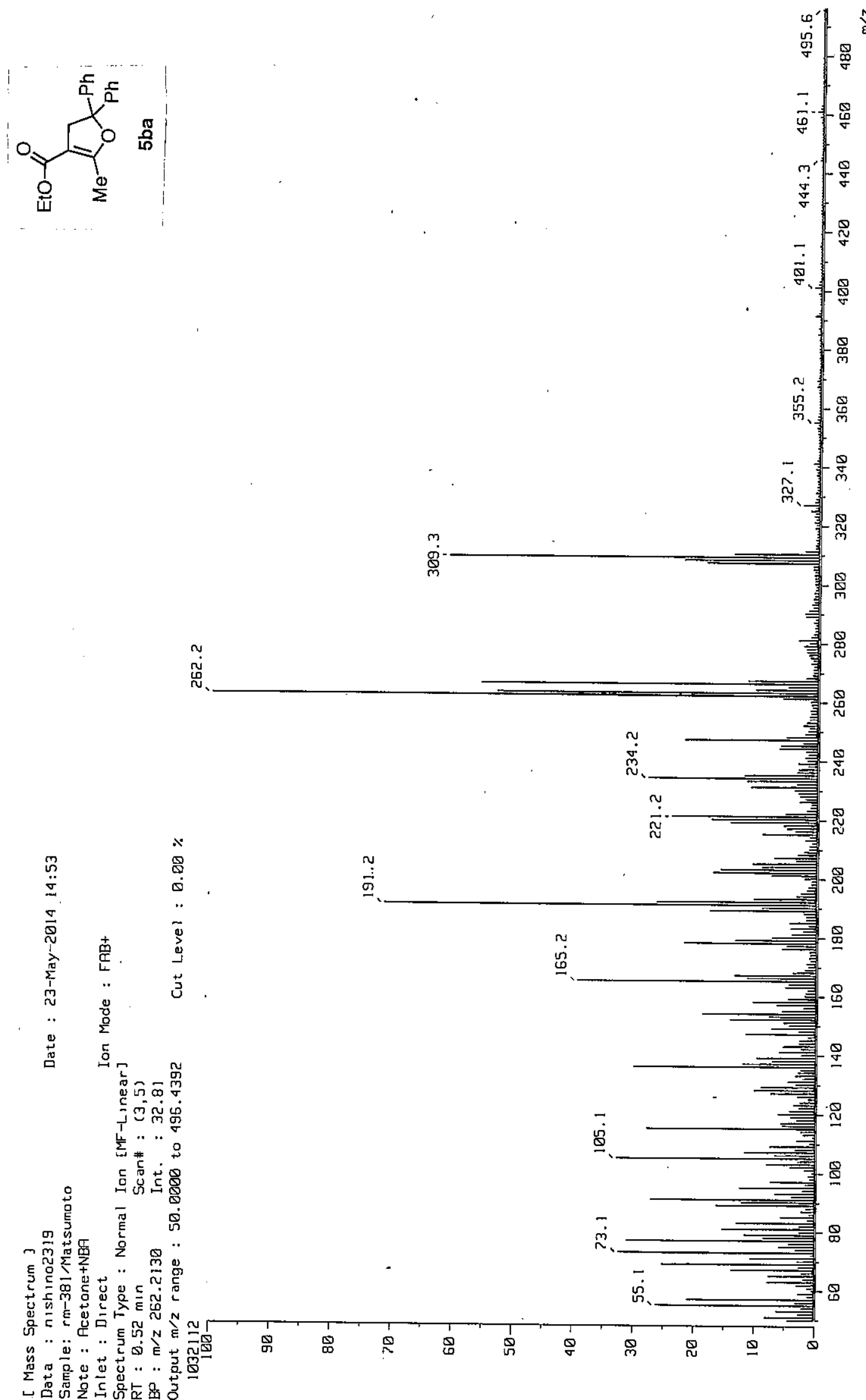


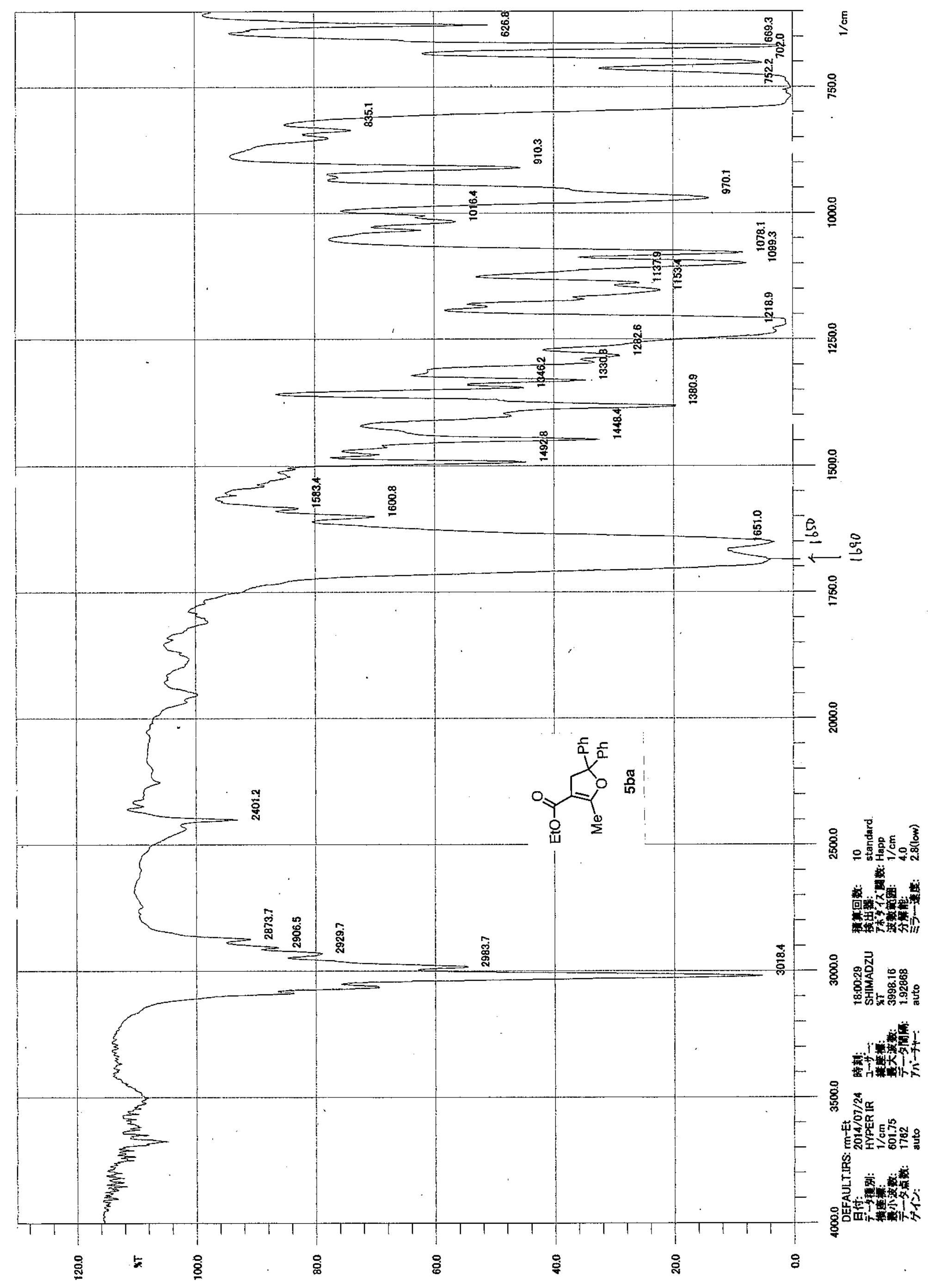




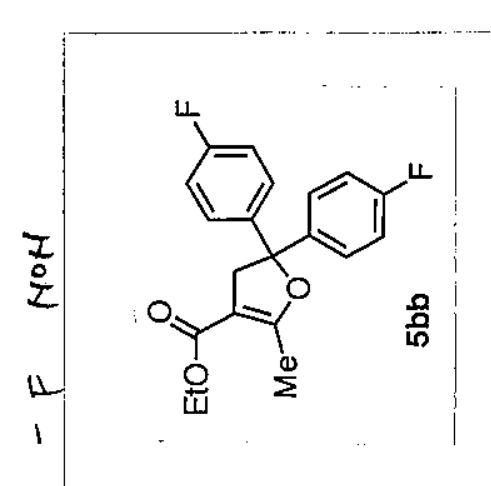


<smiles>CC1=C(C([O])=O)CC(c2ccc(C)cc2)(c2ccc(C)cc2)O1</smiles>

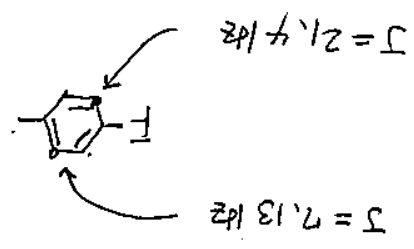




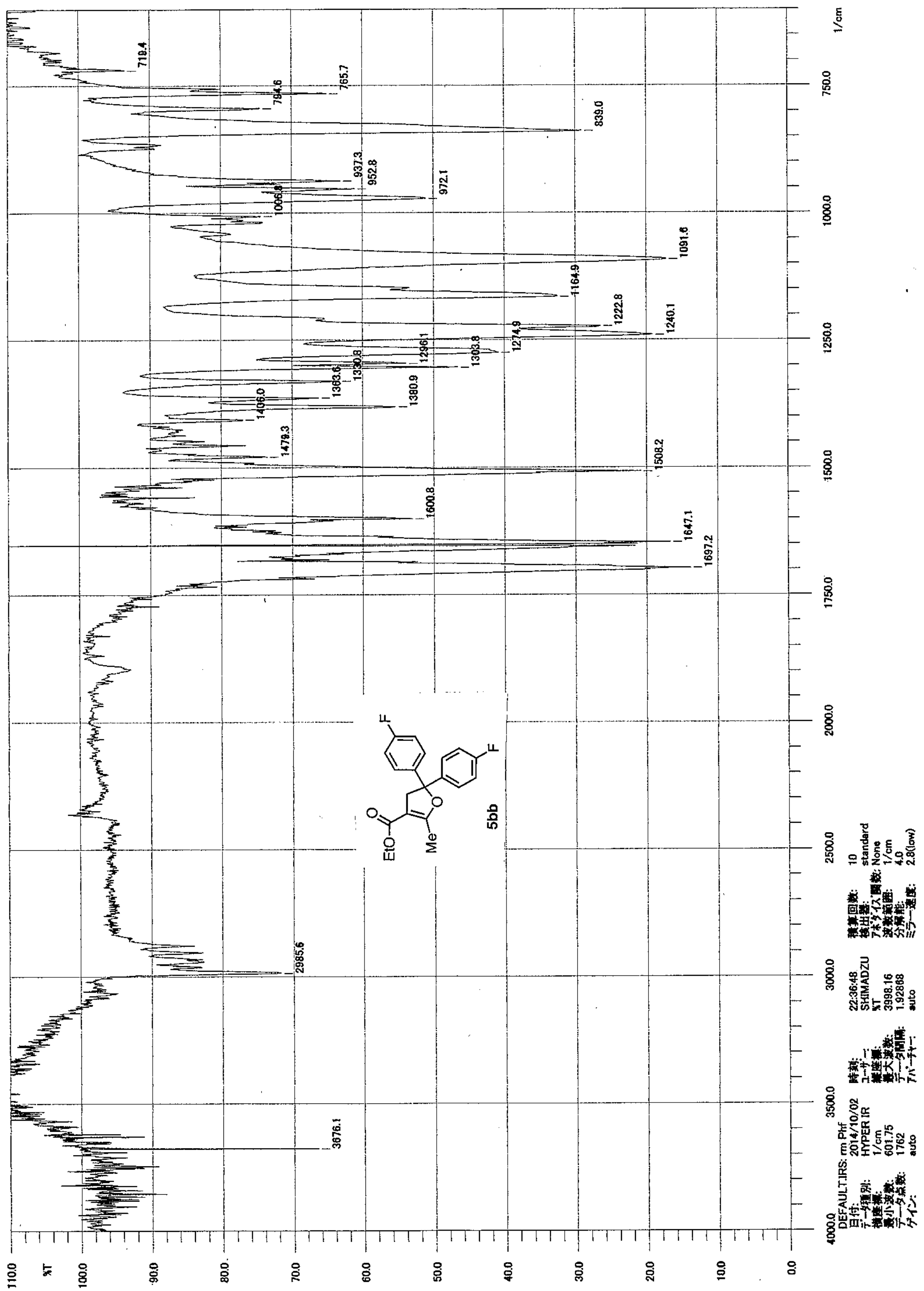




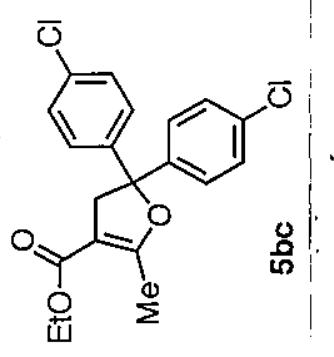

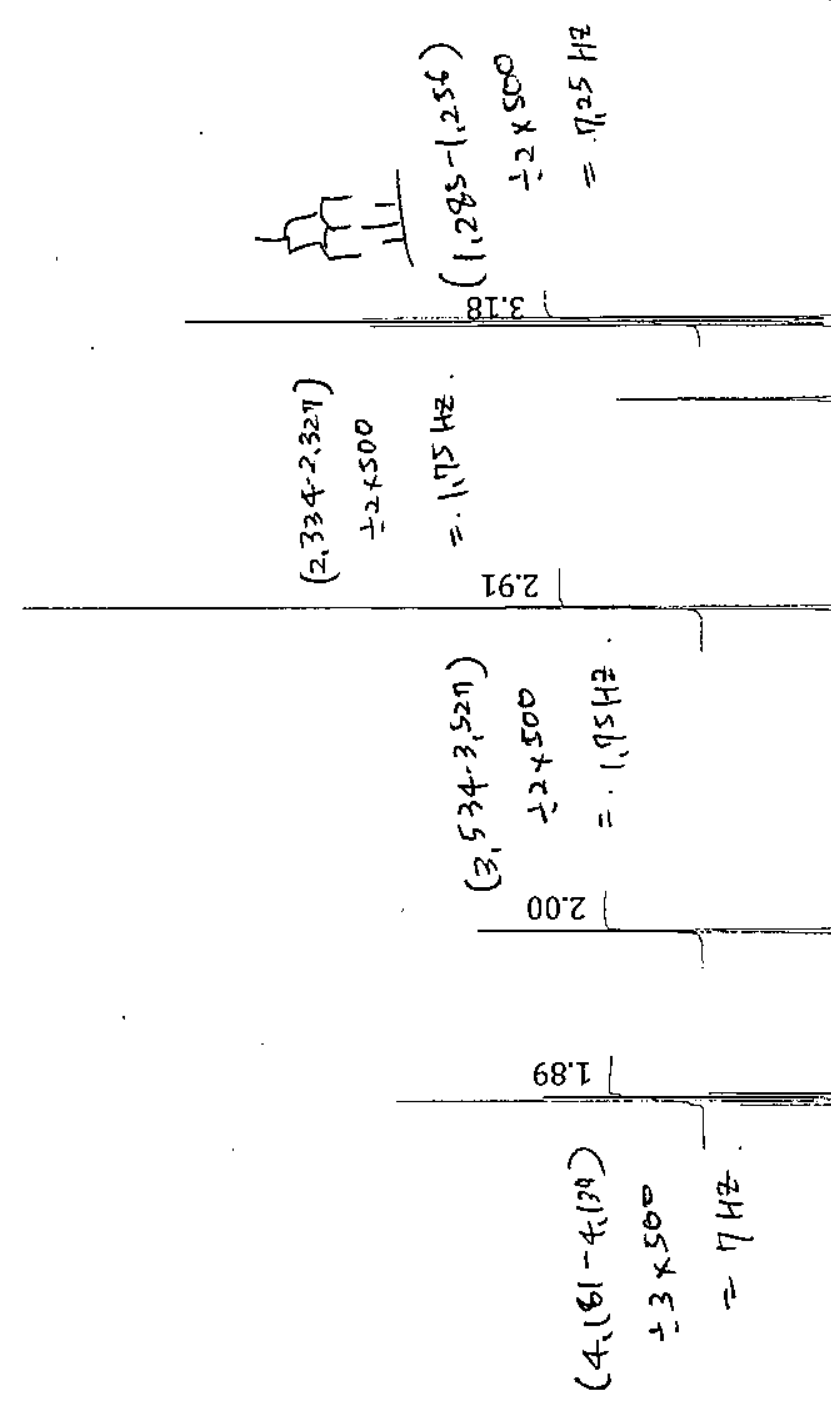

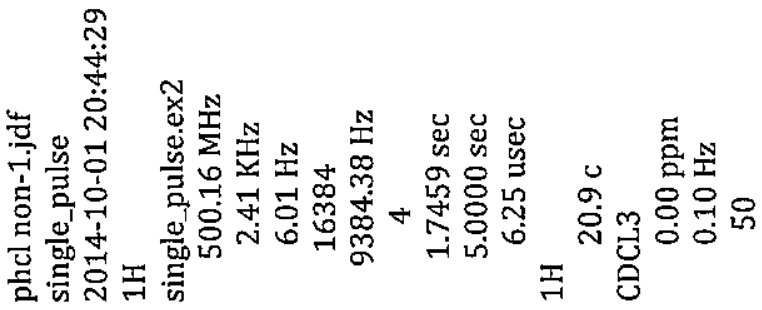

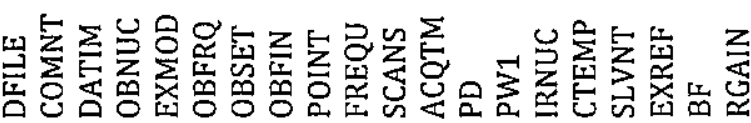




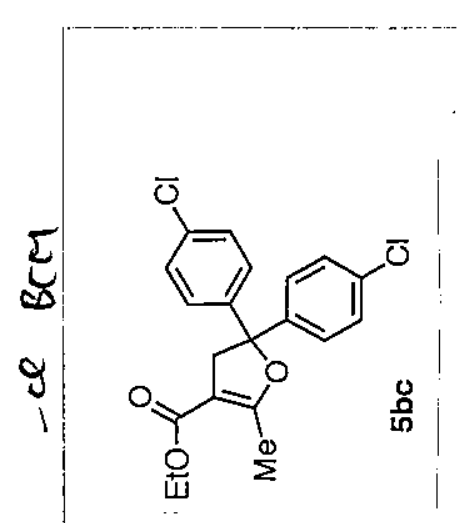

โรL'

$00 \nabla^{\circ}+\mathrm{I}$

$\angle 00^{\circ} \nabla$

in

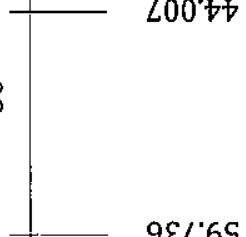

$9 \varepsilon L ' 6 S$

2SL'9L

$000^{\circ} \mathrm{LL}$

8SZLL

6St06

8Z8ㄷㄴ

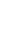

$8+0 \angle Z I$

E6G'8ZT.

$899^{\circ} \varepsilon \varepsilon \tau$

SIZ $\varepsilon \pitchfork I$

윰

는

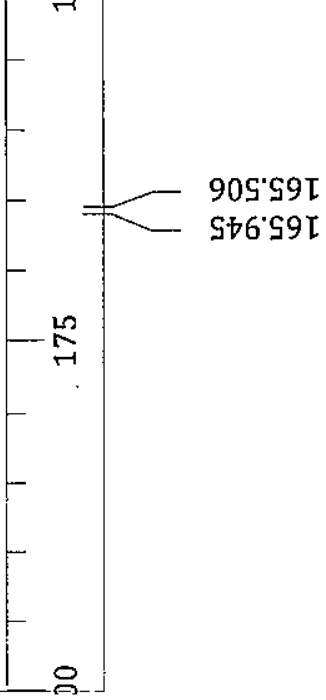


(1)

$\varepsilon Z I \div I$

ZSE'D

$6 \mathrm{~S} 6^{\circ} \&$

은

욱

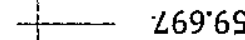

$\angle 69^{\circ} 6 S$

066'92.

StS'8ZT 


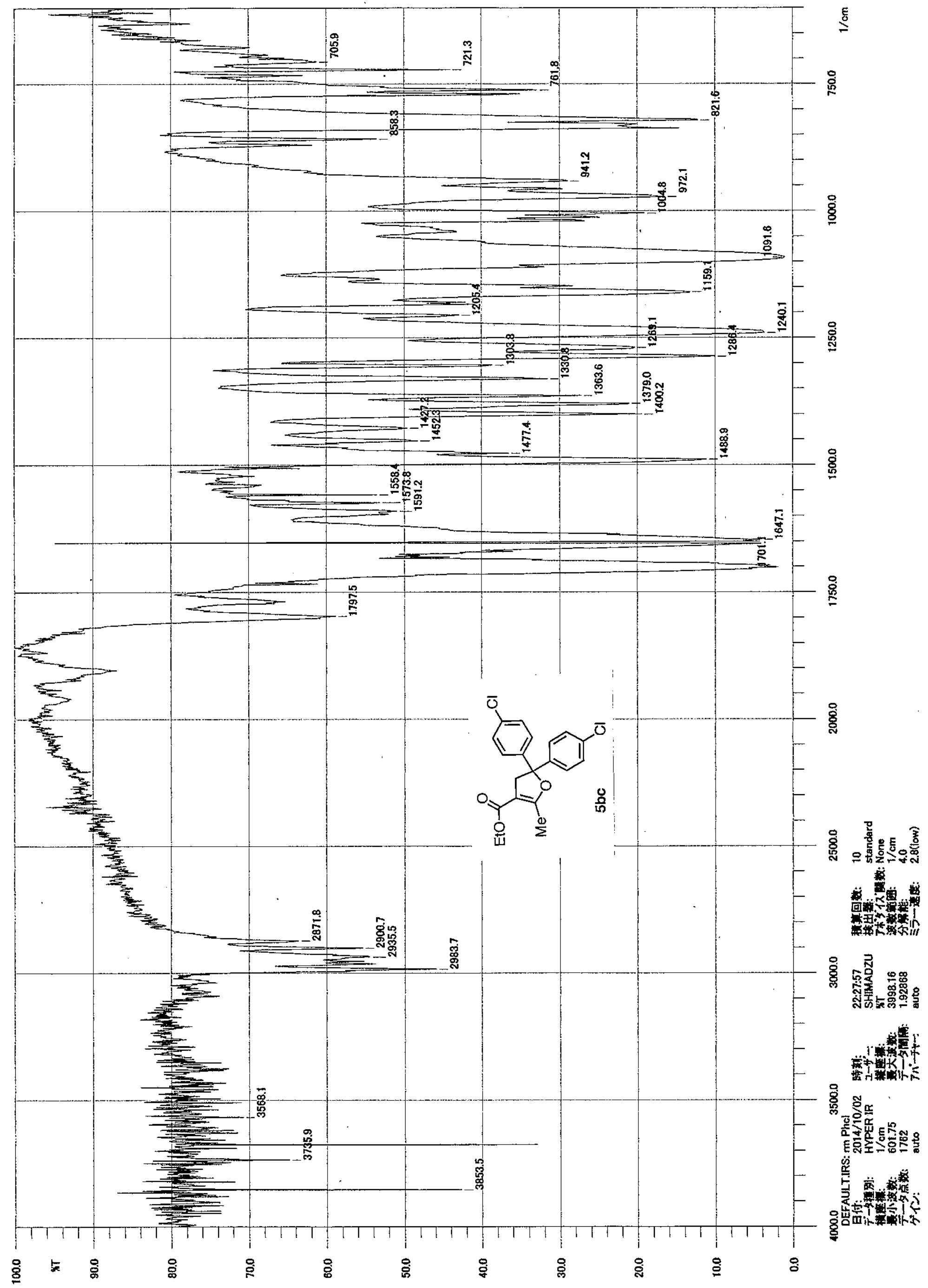



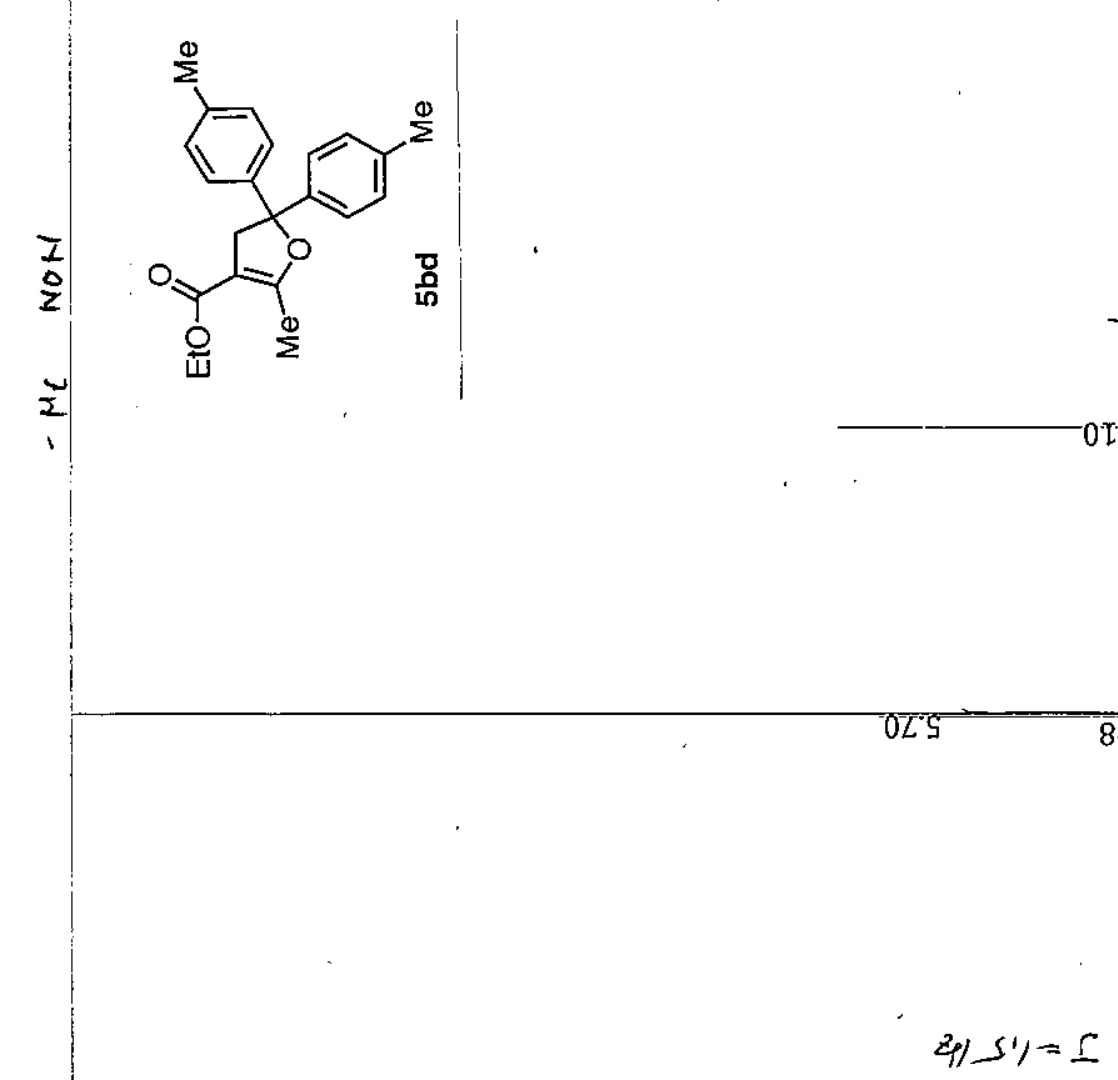

867

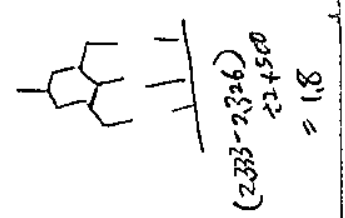

$\underline{2}$

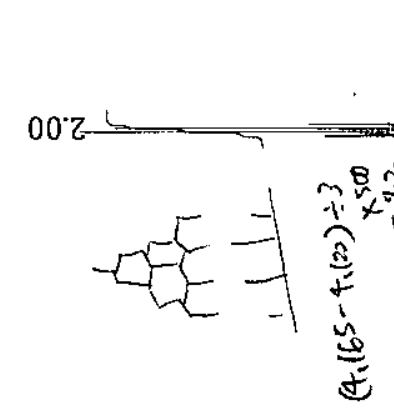




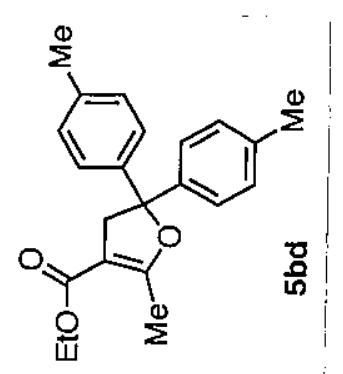



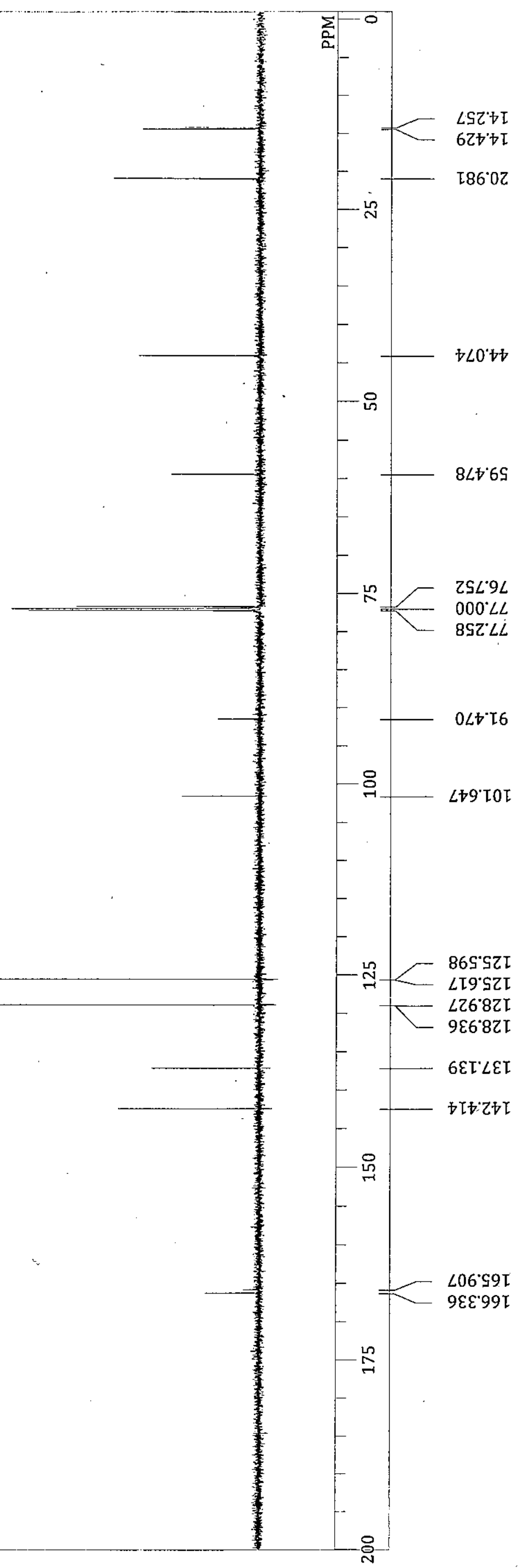



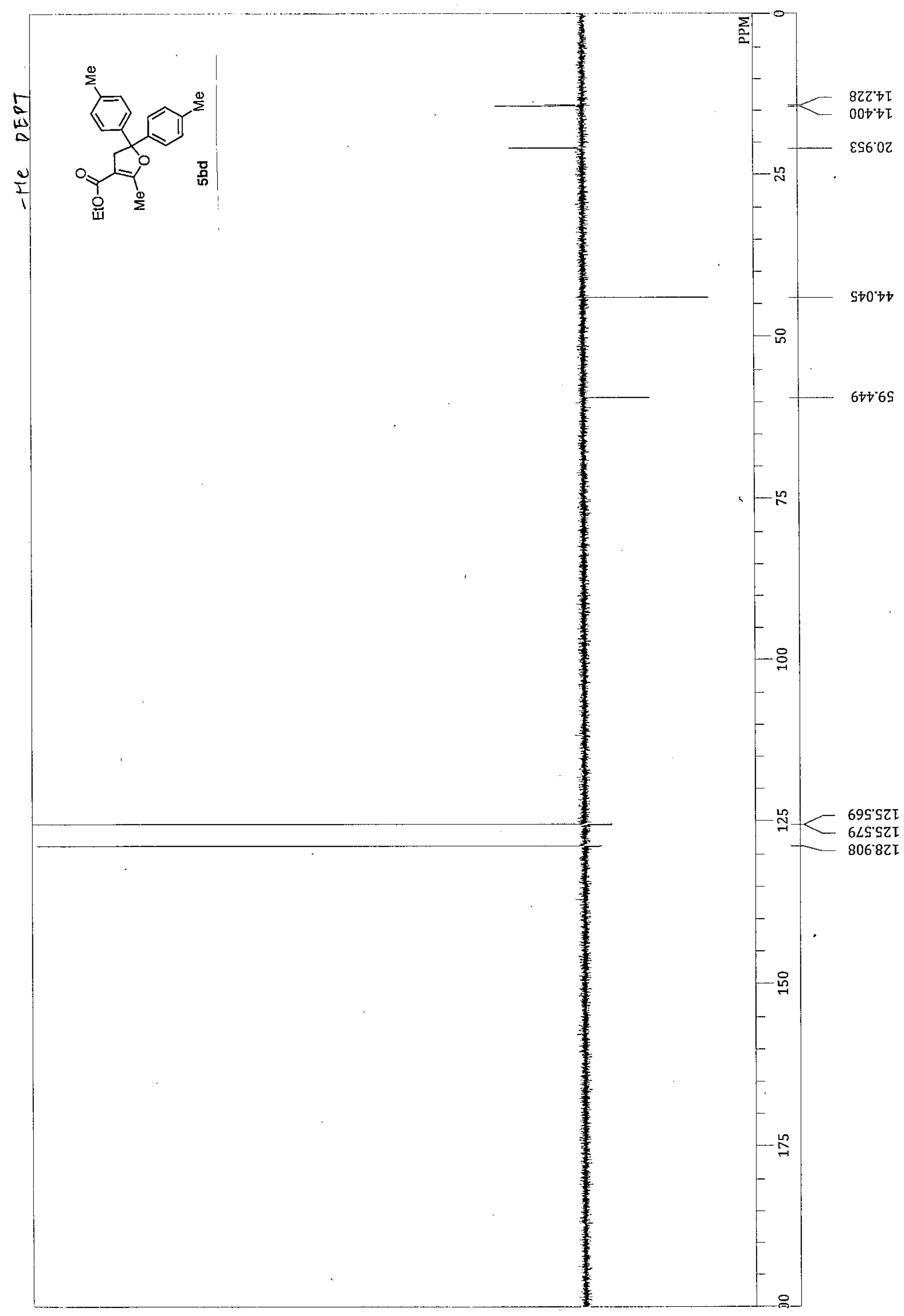


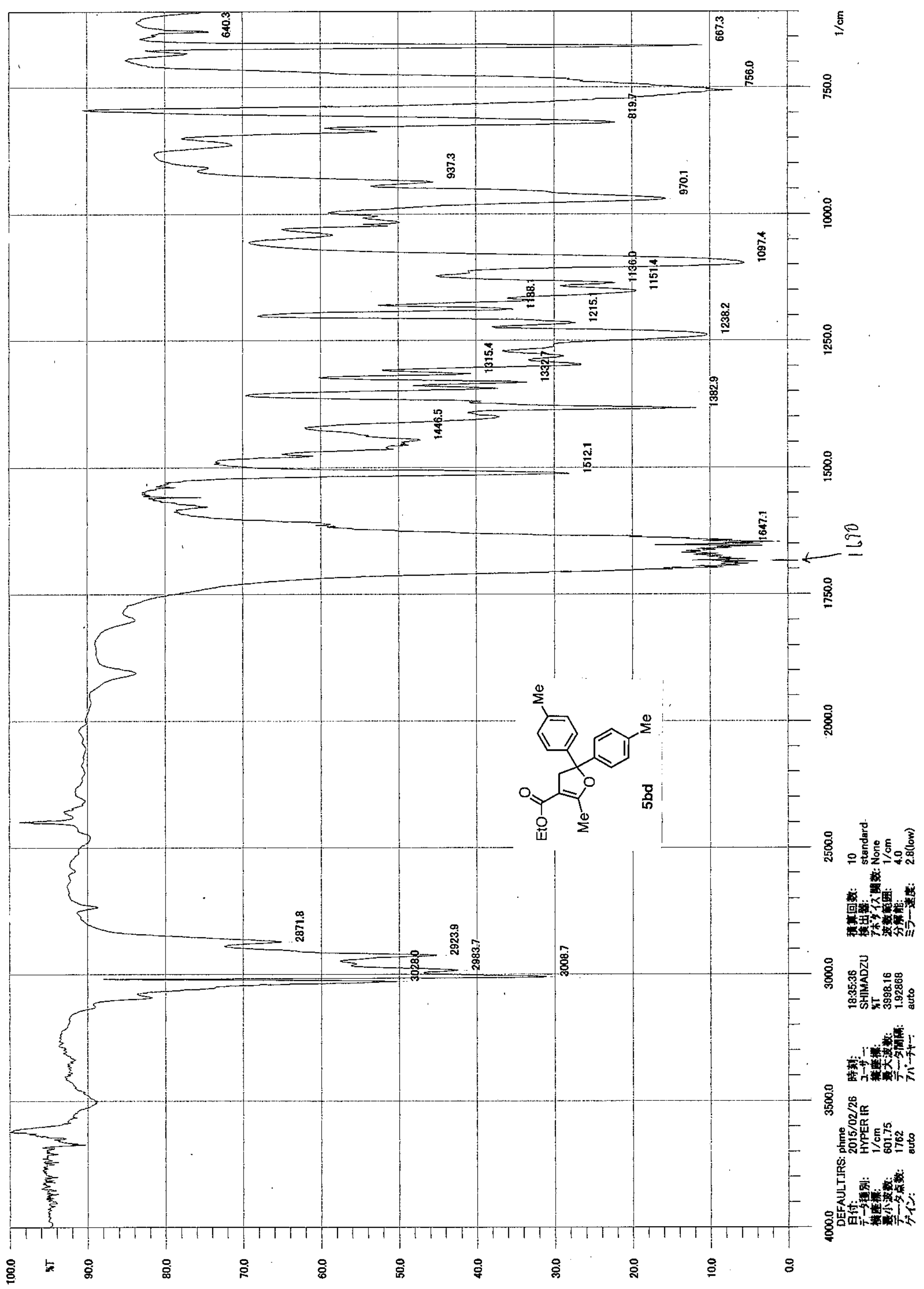




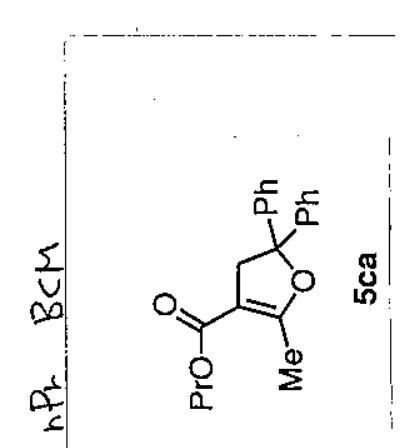




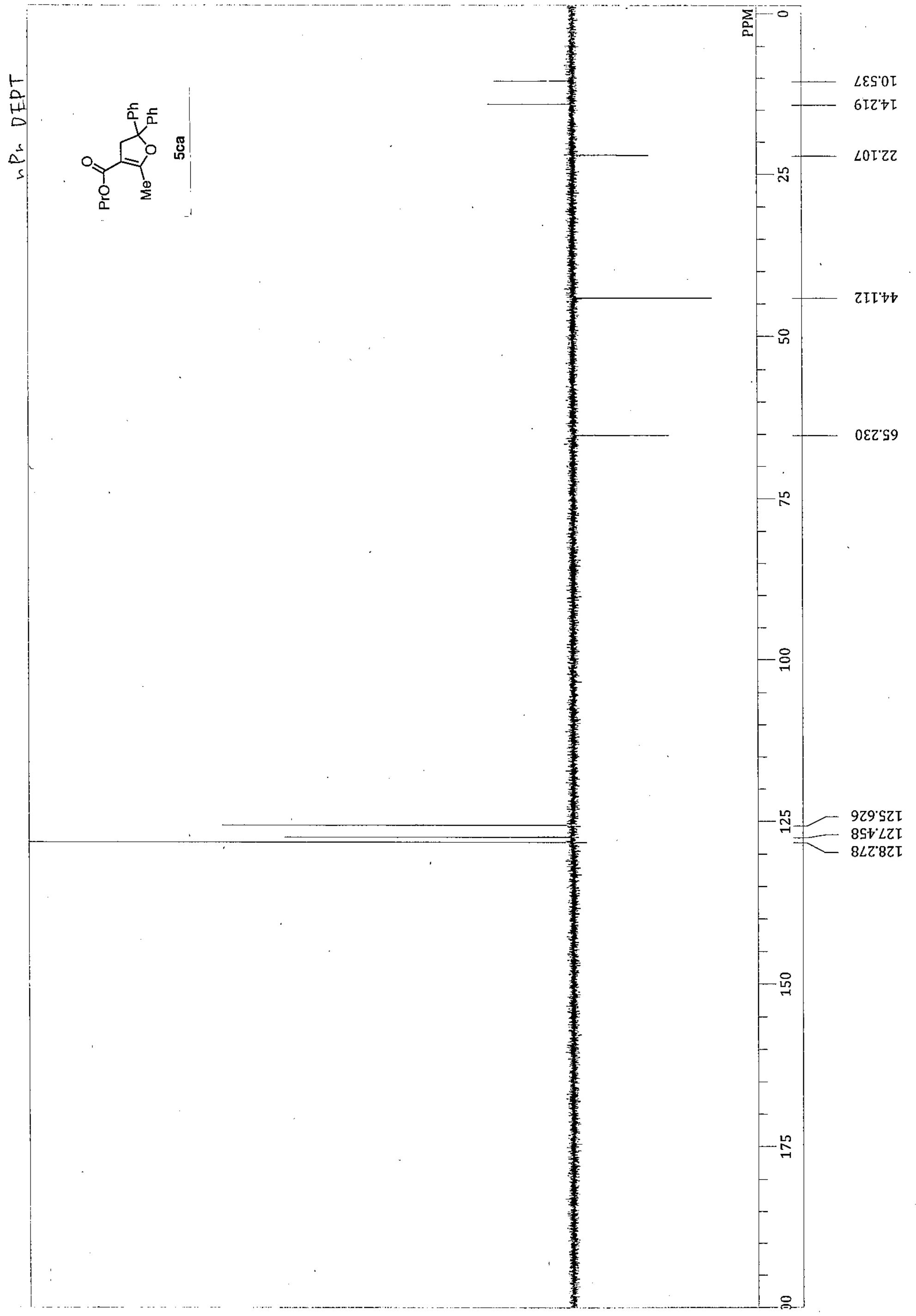




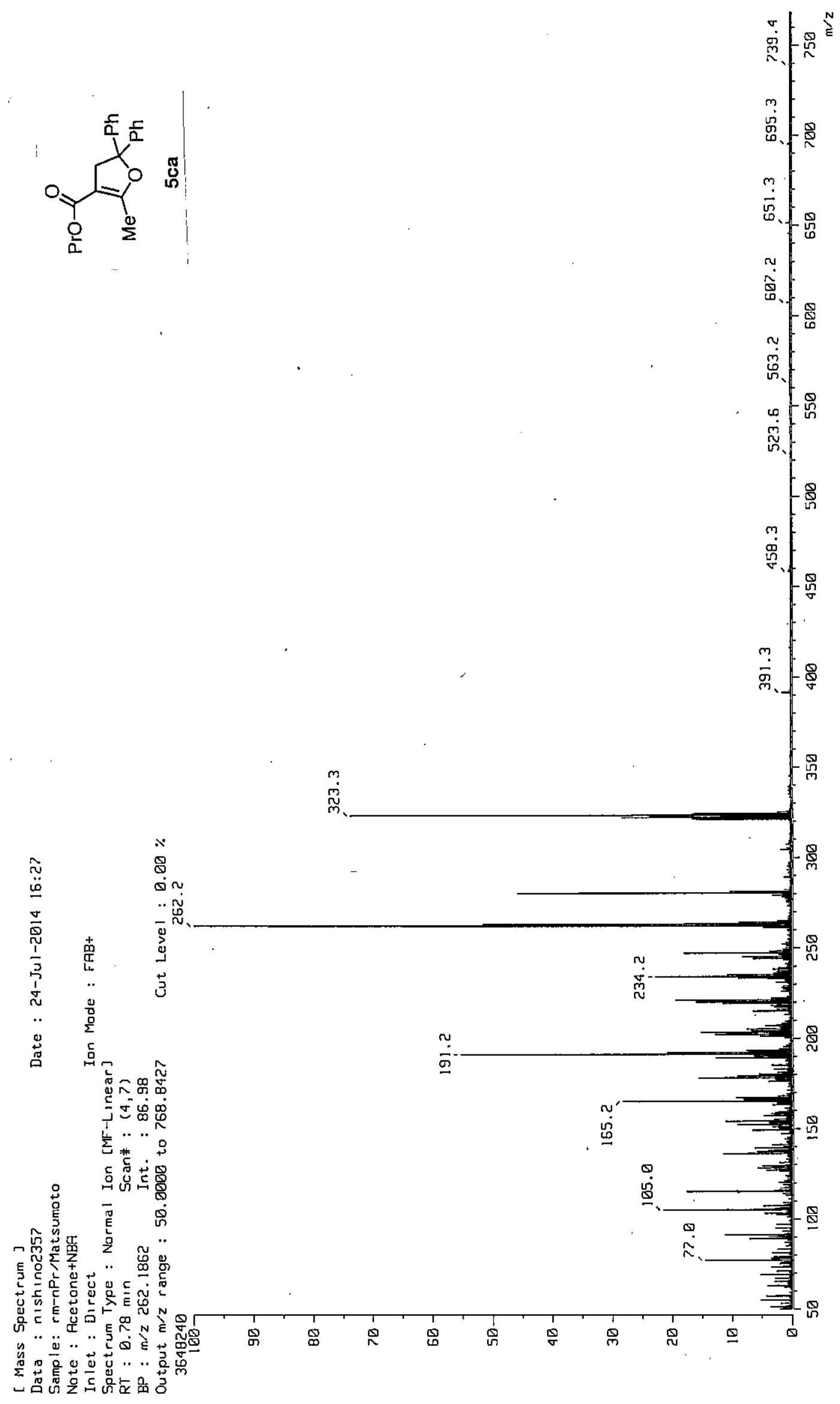




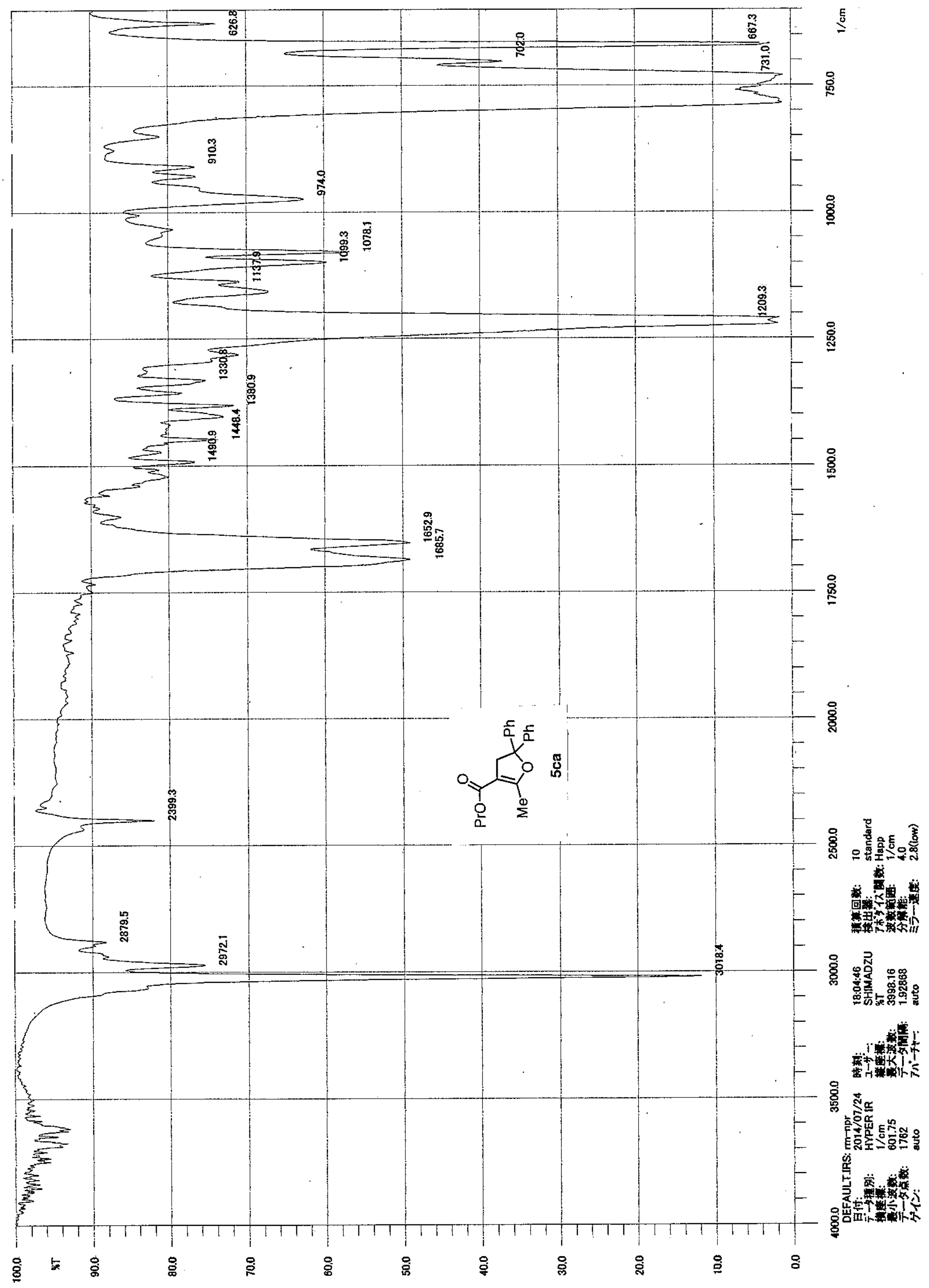




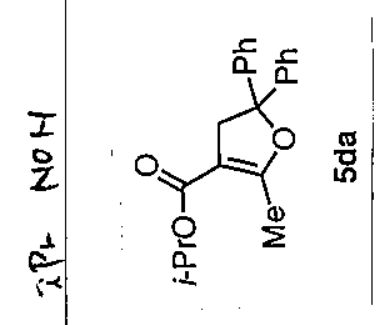


<smiles>COC(=O)C1=C(O)O[C@](C)(C(C)(C)C)C1</smiles>

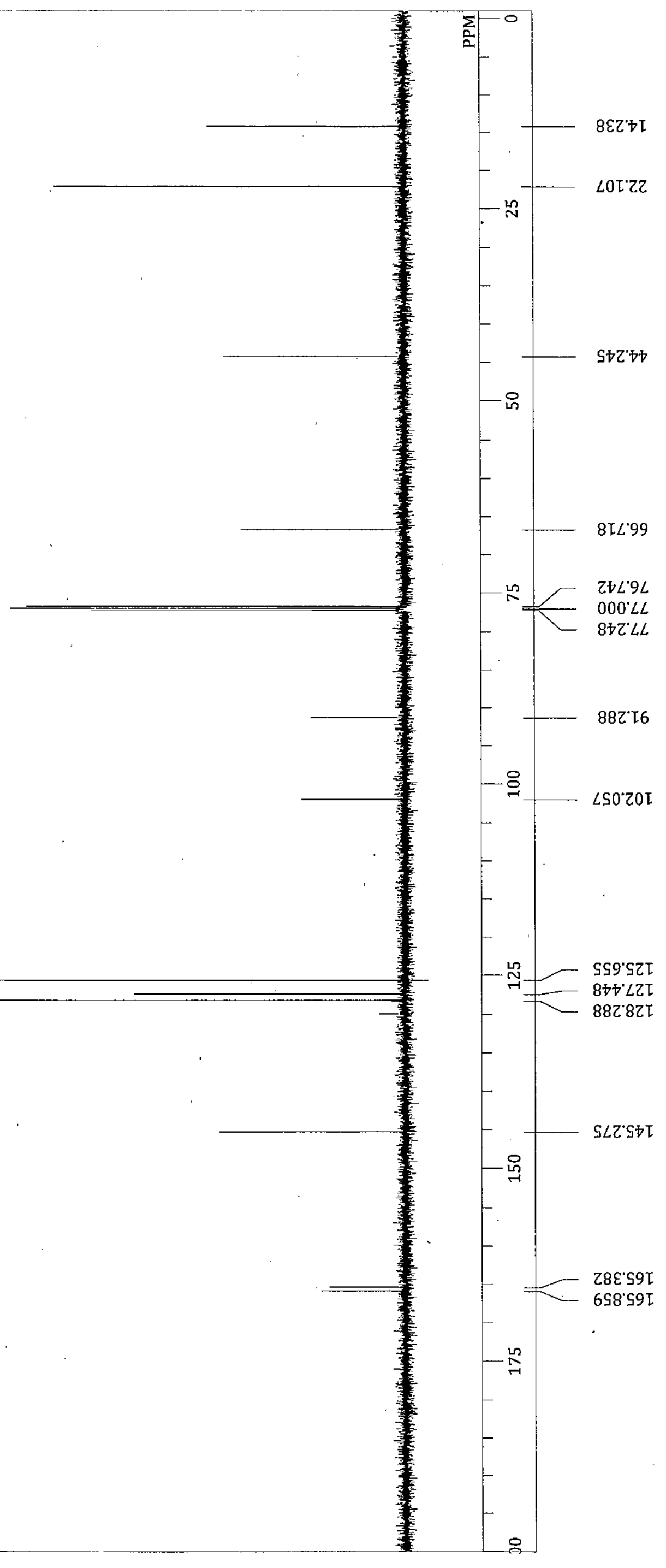




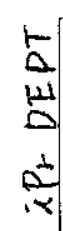
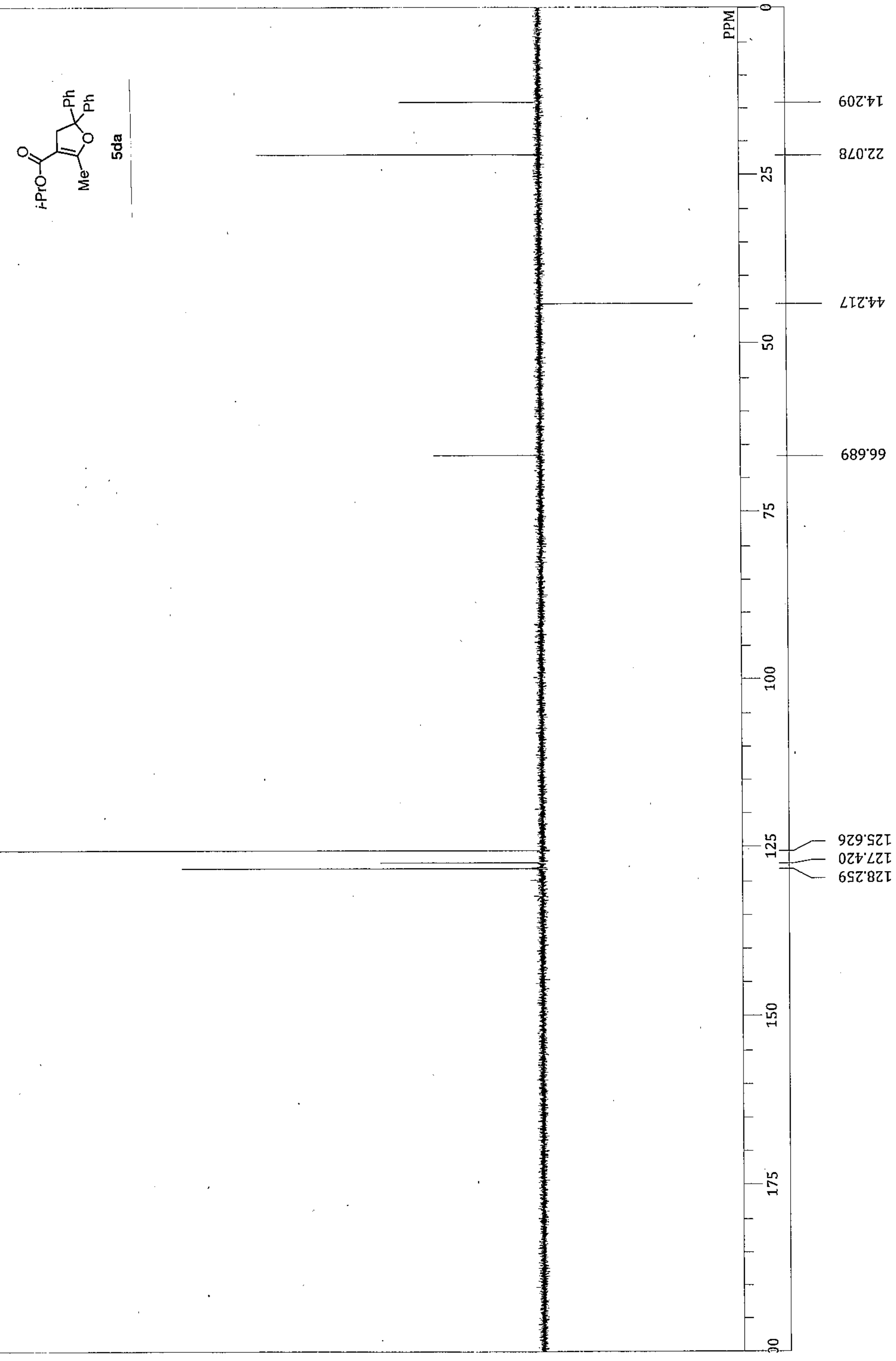

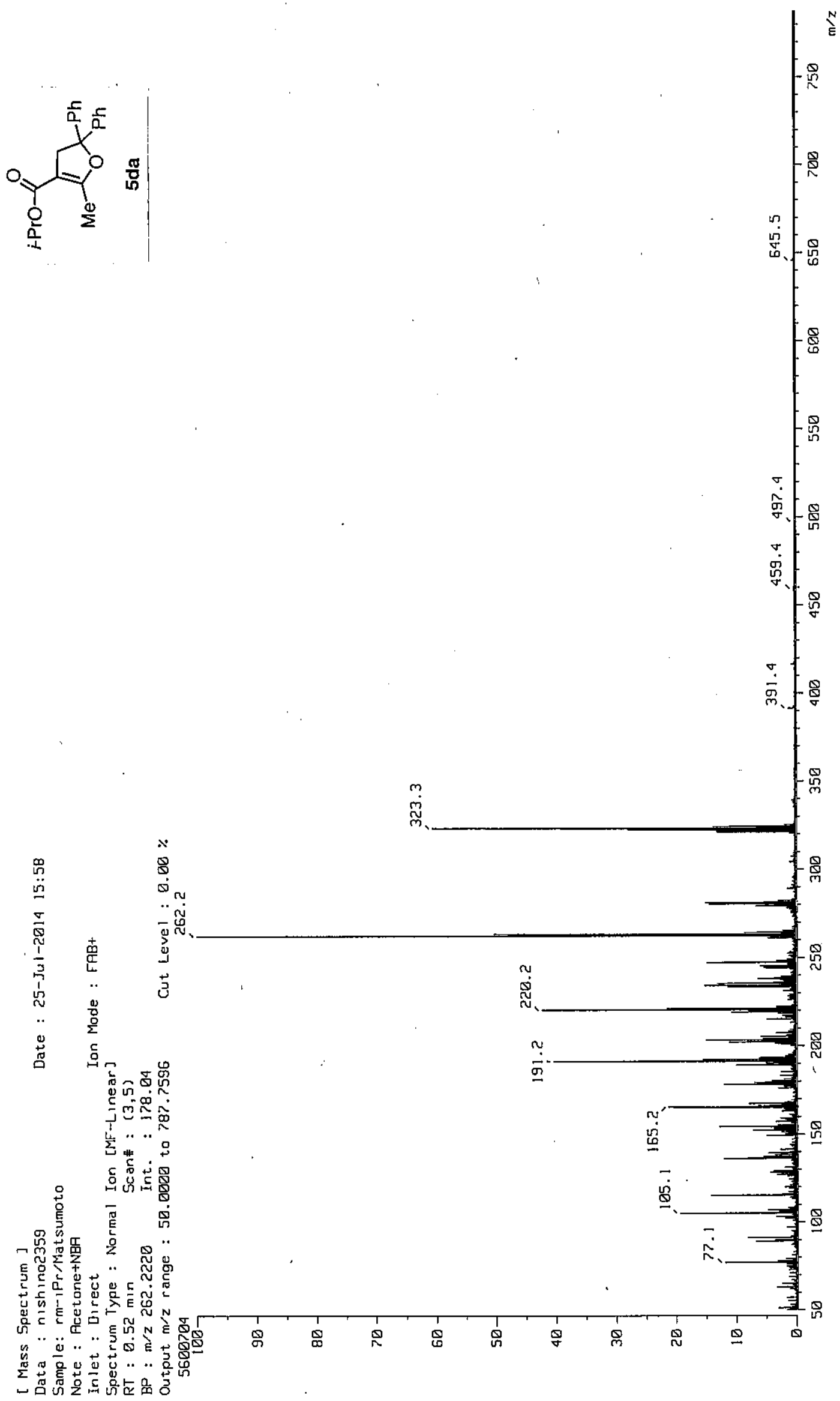


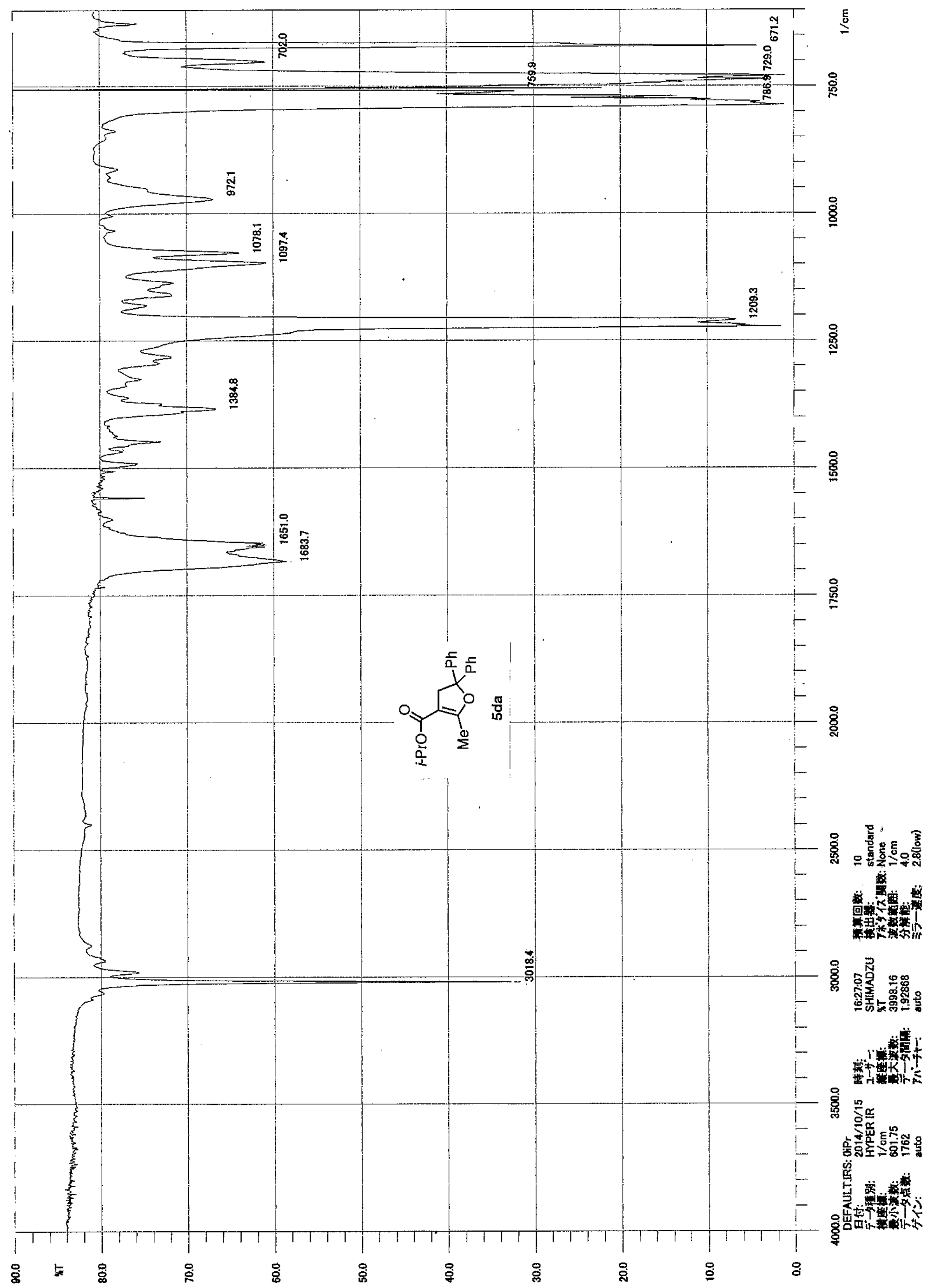


<smiles>[CH]C(=O)C1=C(O)OC(C)(C)C1</smiles>

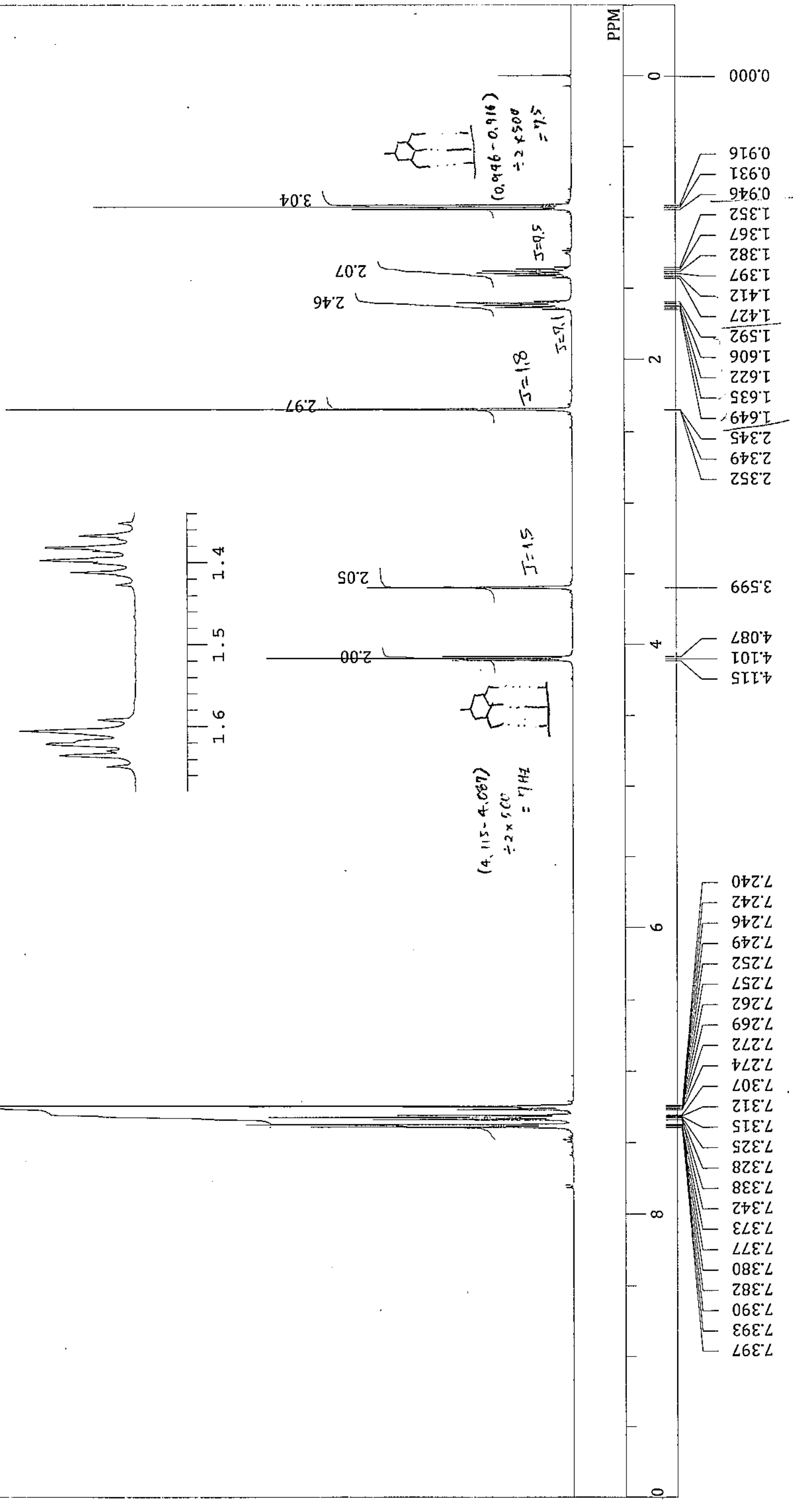




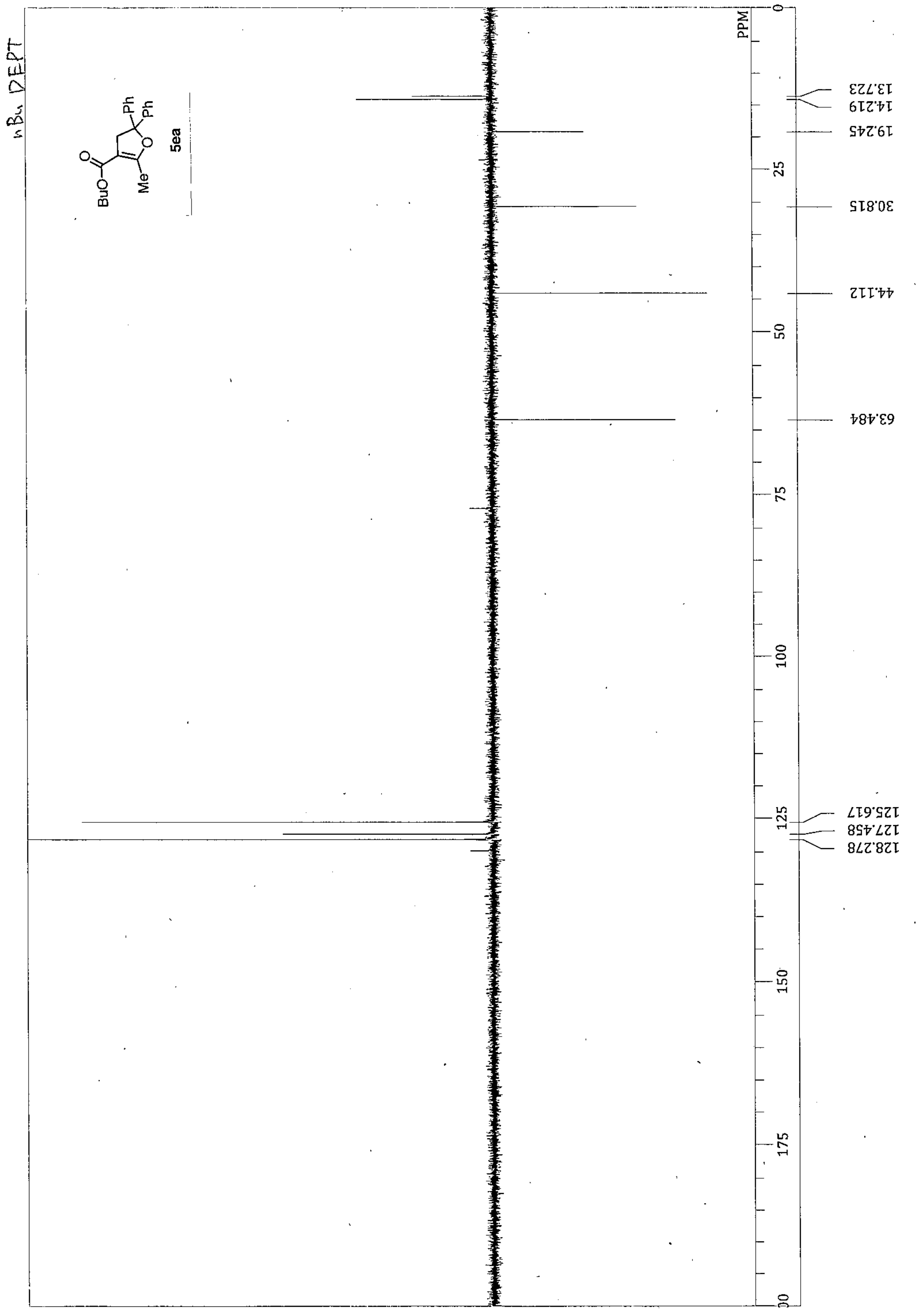



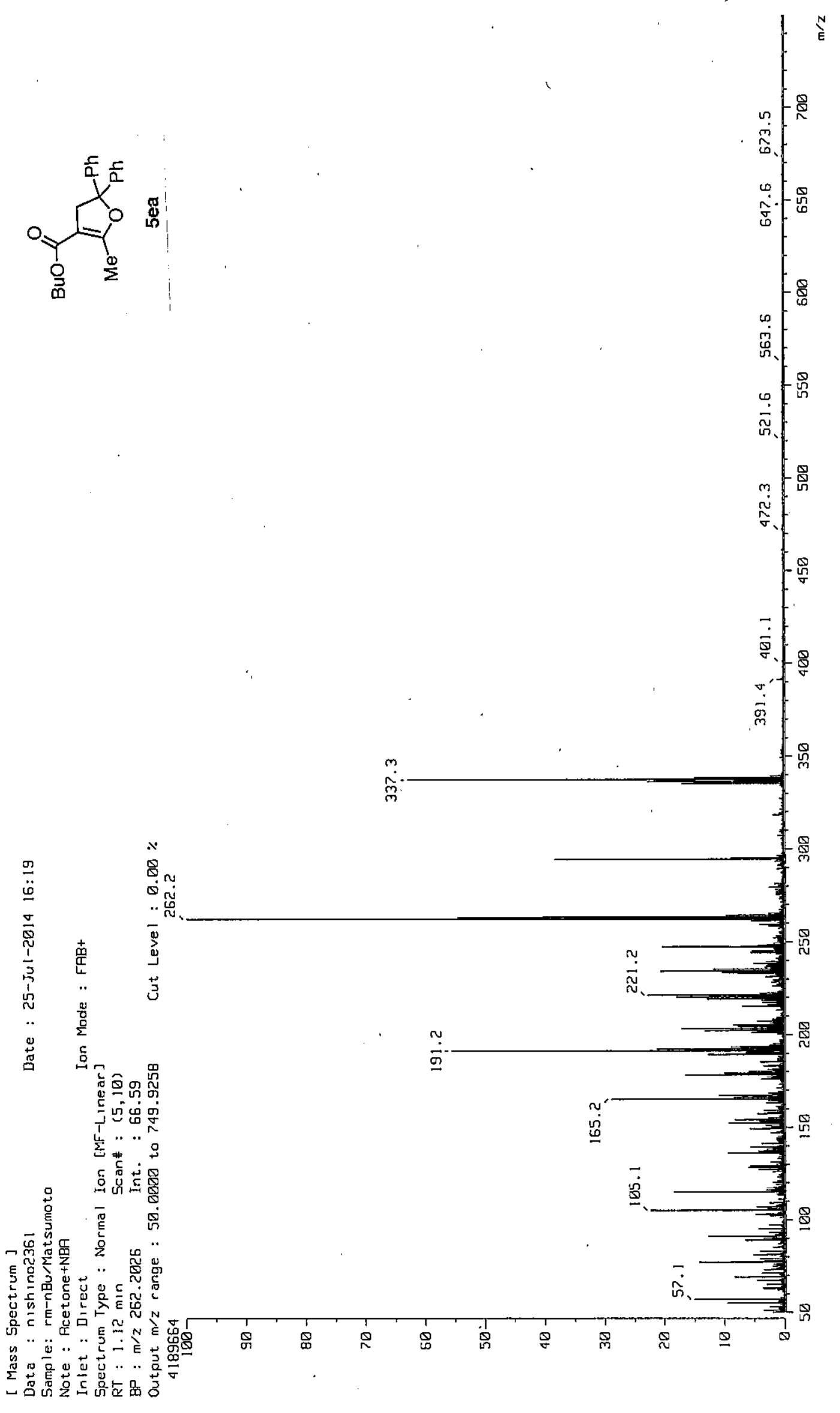


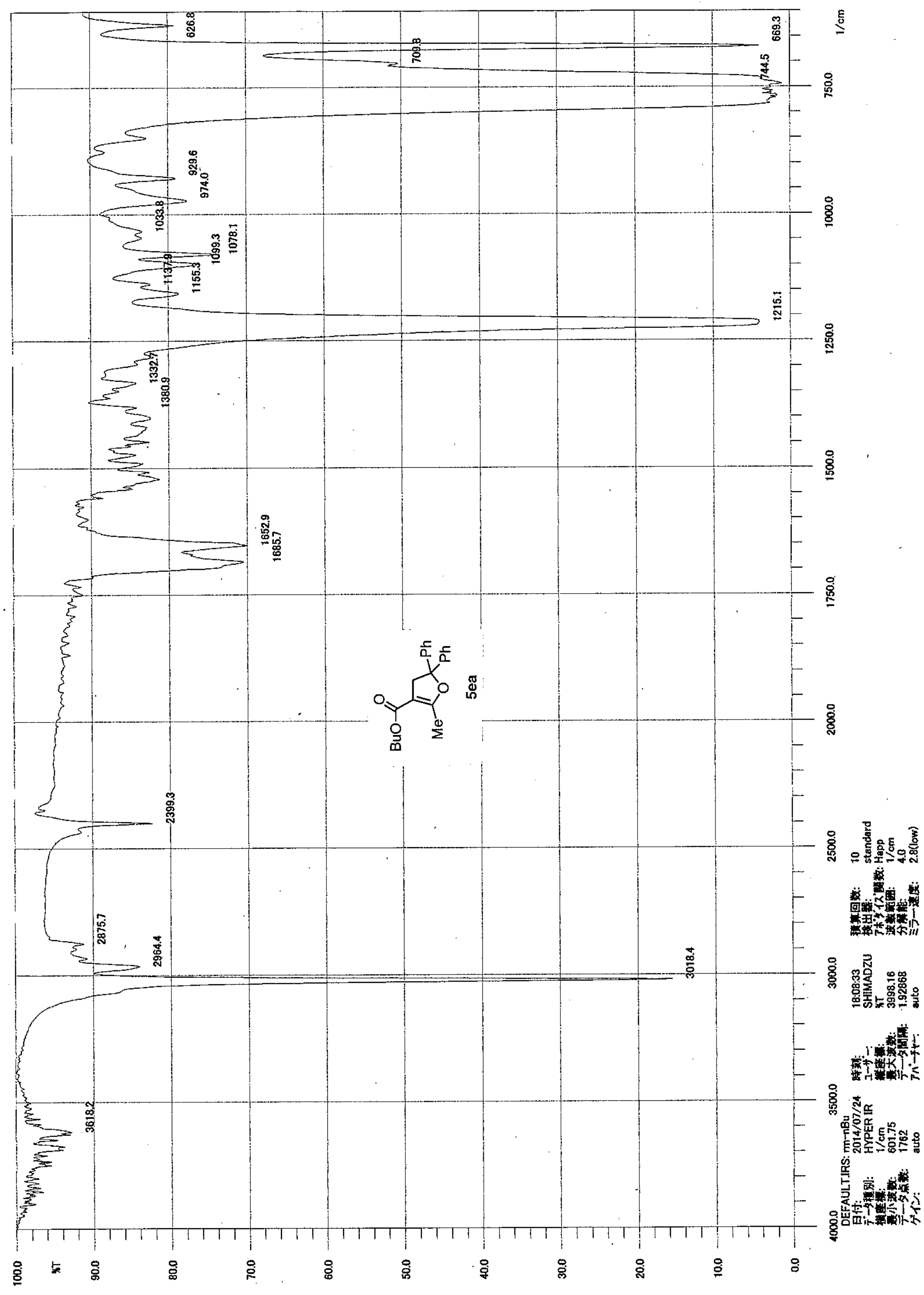


<smiles>CC1=C(C(=O)O)CC(C(F)(F)F)(C(F)(F)F)O1</smiles>

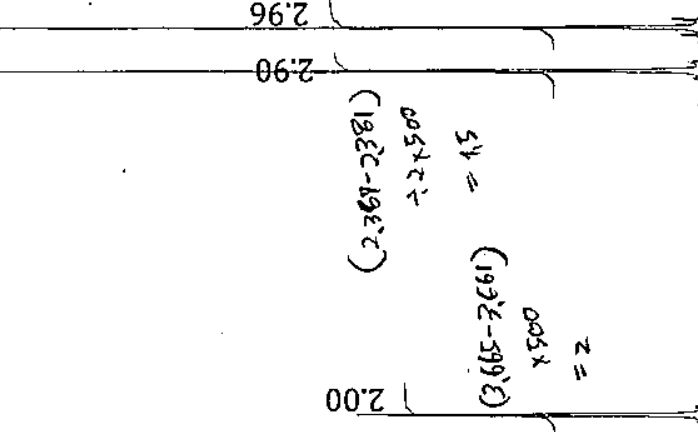




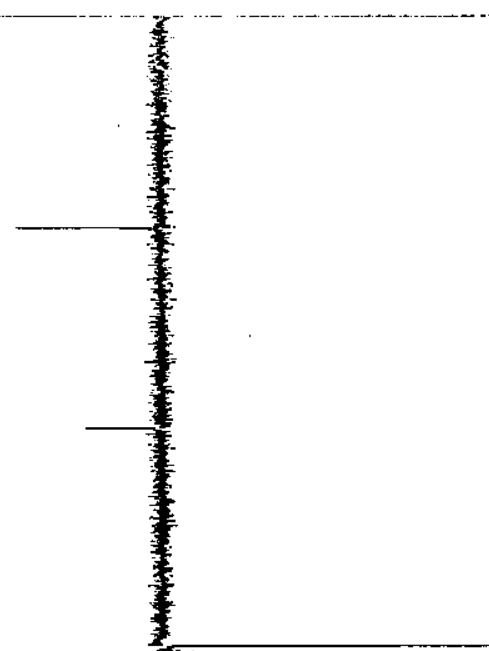

$-0$

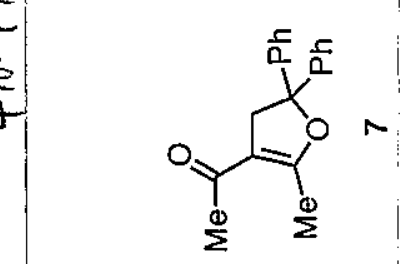

$\downarrow t I \cdot S T$ 


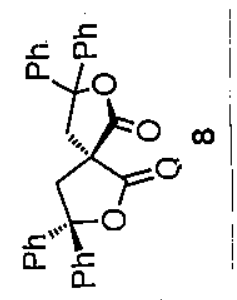

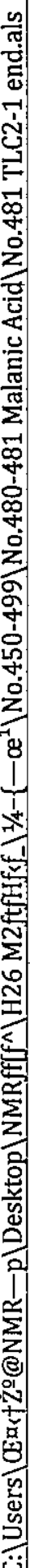

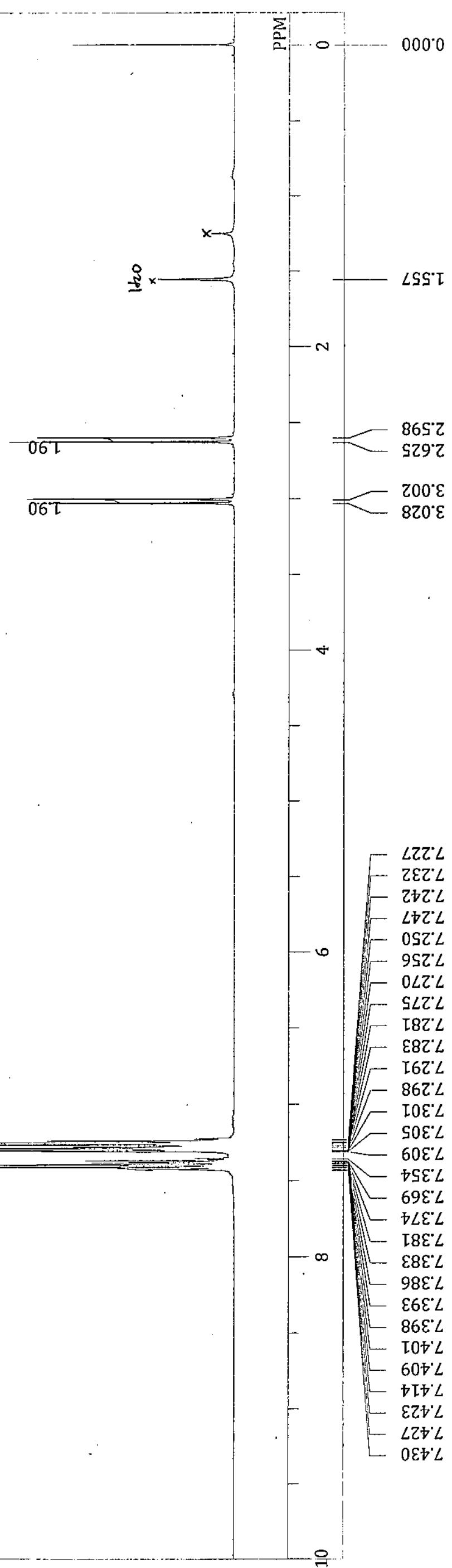



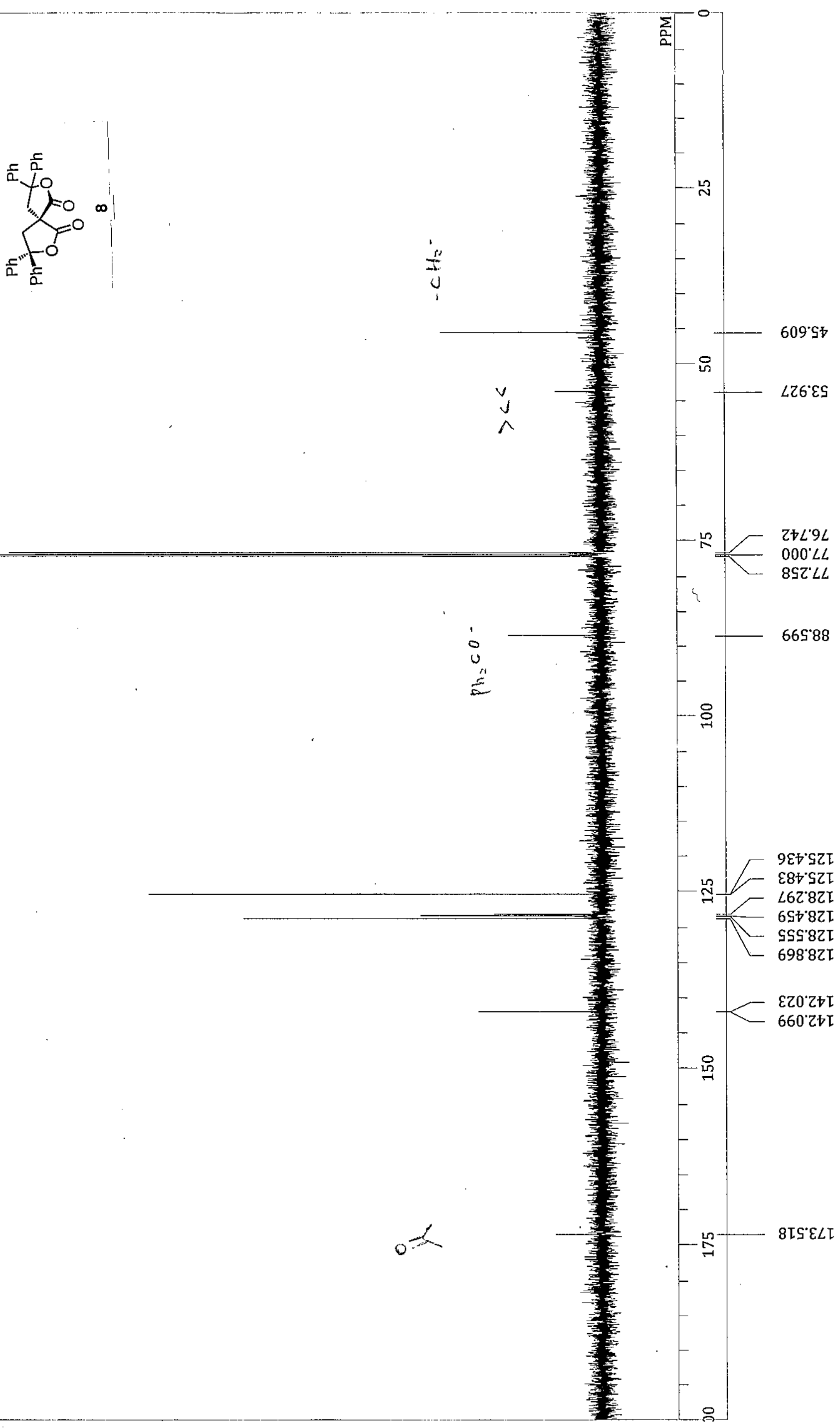

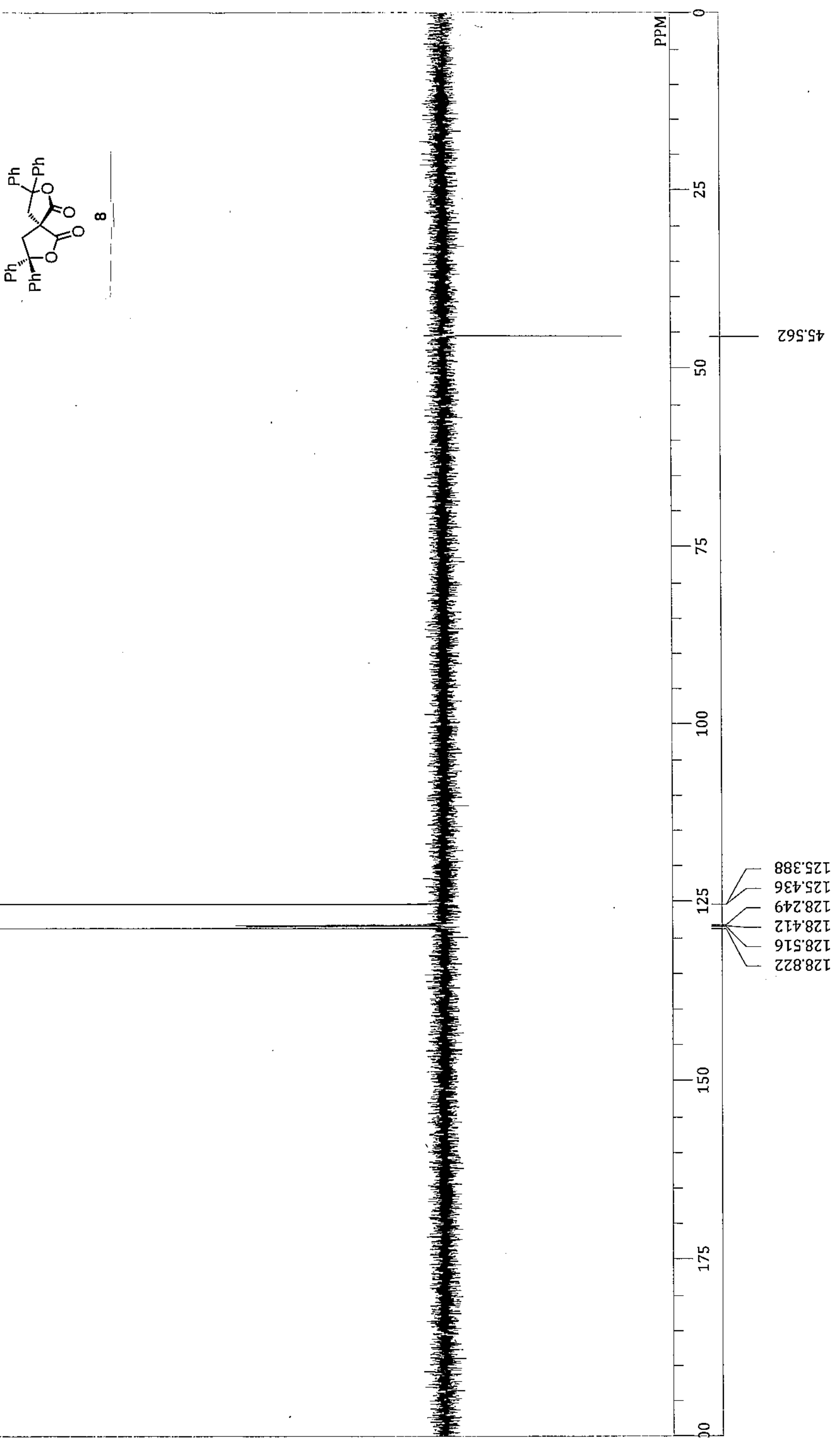


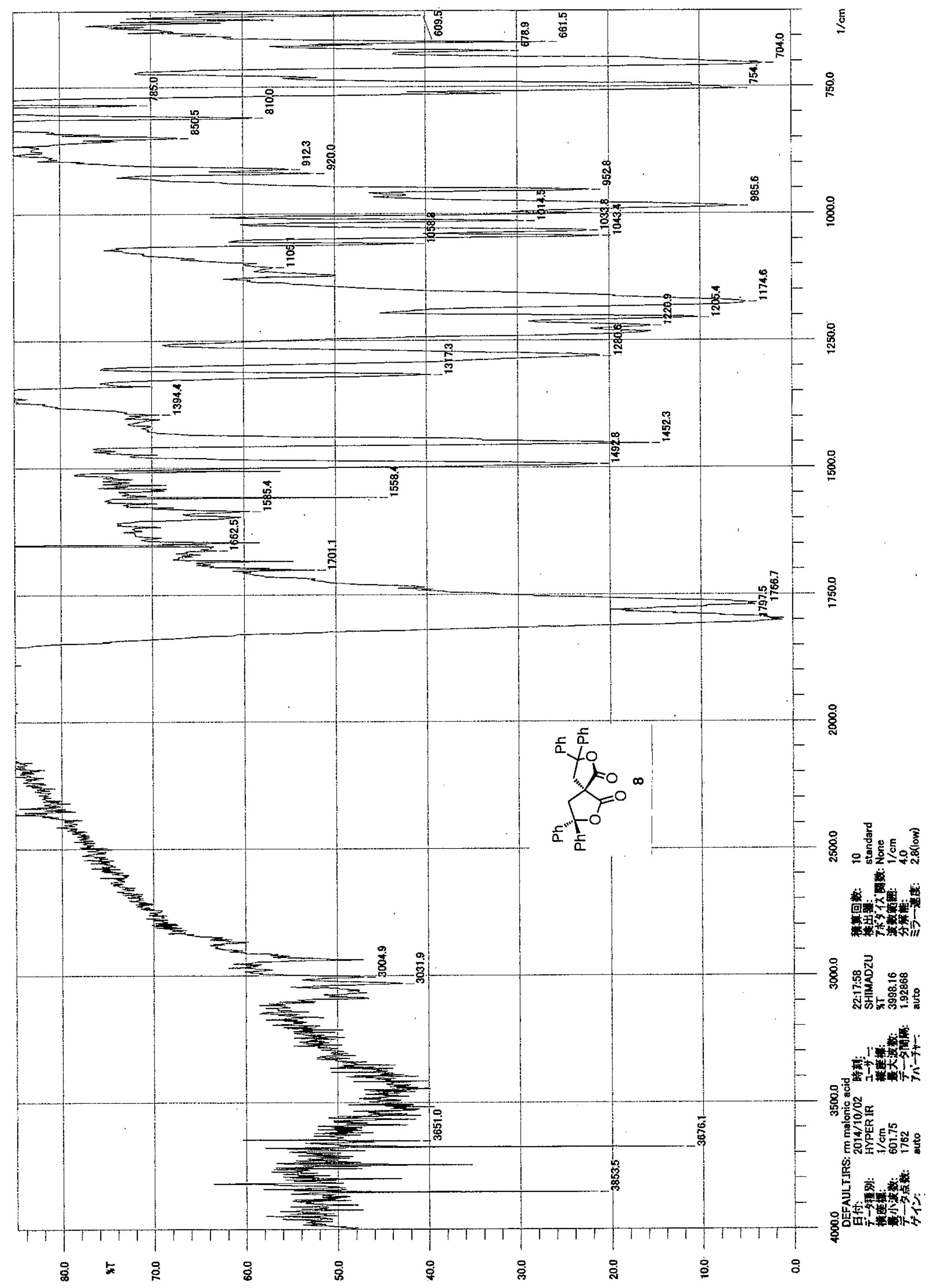




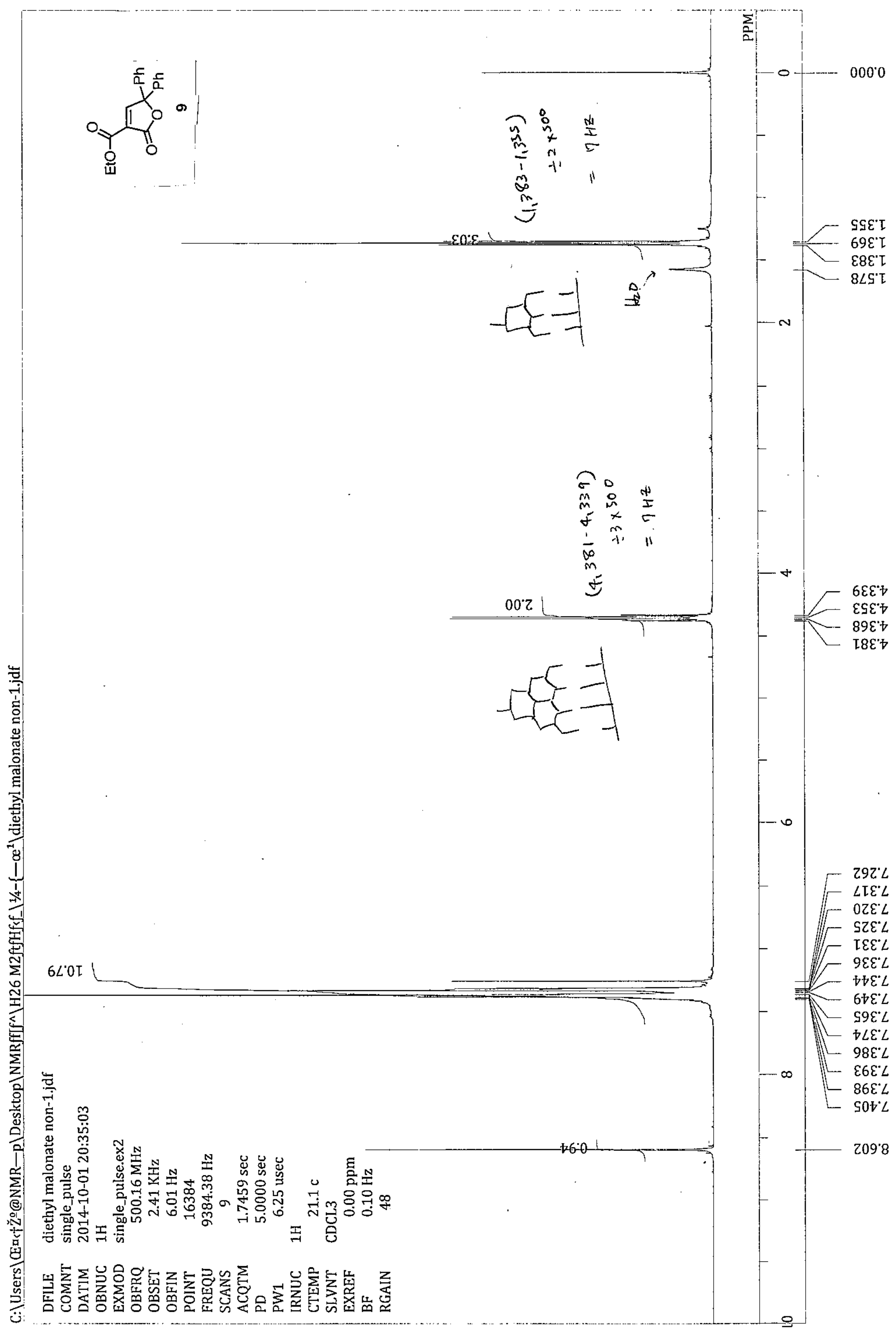


<smiles>[CH]C(=O)C1=C[C@]([CH2-])(C)OC1=O</smiles>

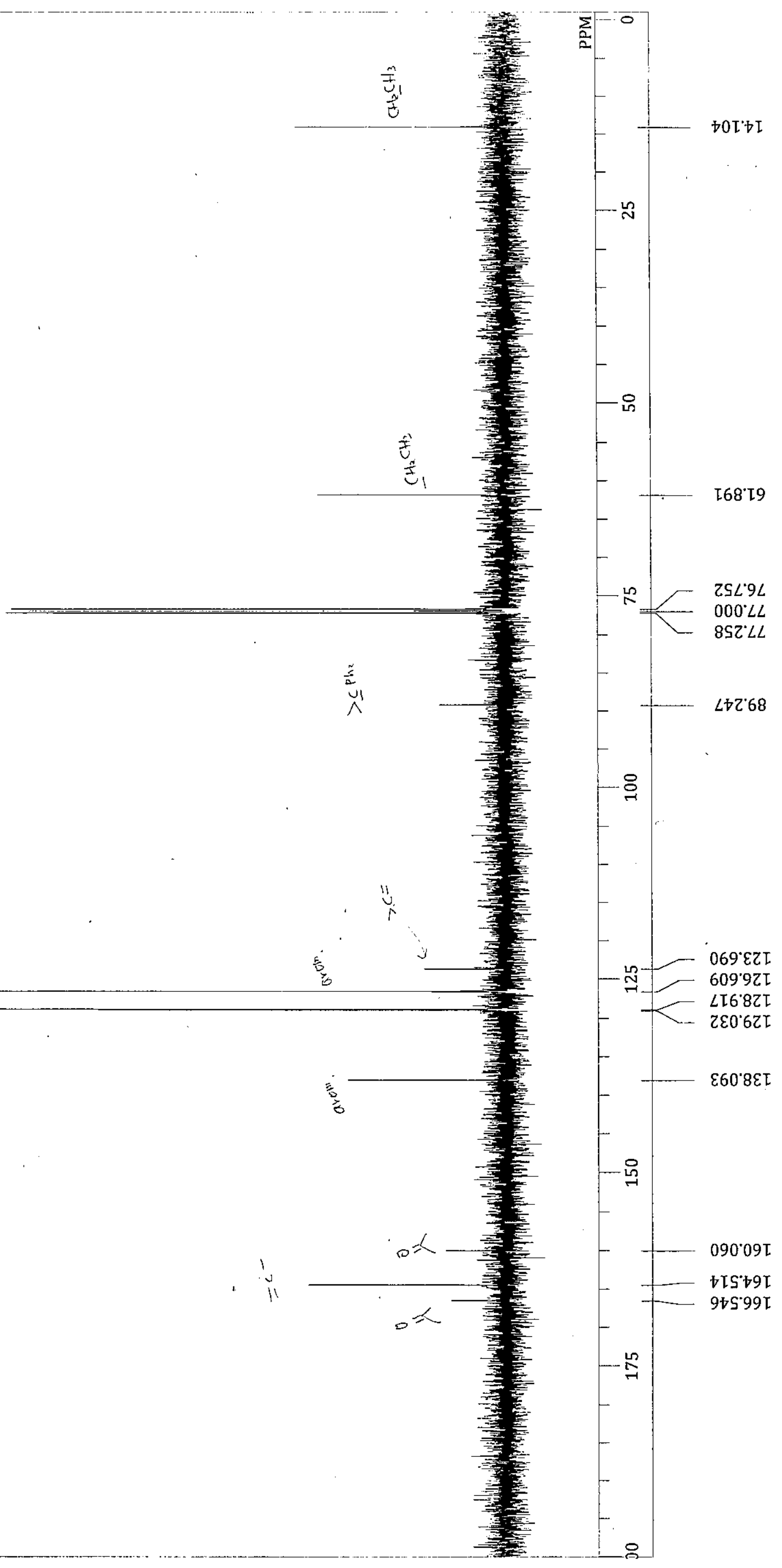


<smiles>[CH]C(=O)C1=C[C@]([CH2-])(C([CH])=O)OC1=O</smiles>

$580^{\circ} \mathrm{T}$

288' 19

$065^{\circ} 92 \mathrm{~L}$ 


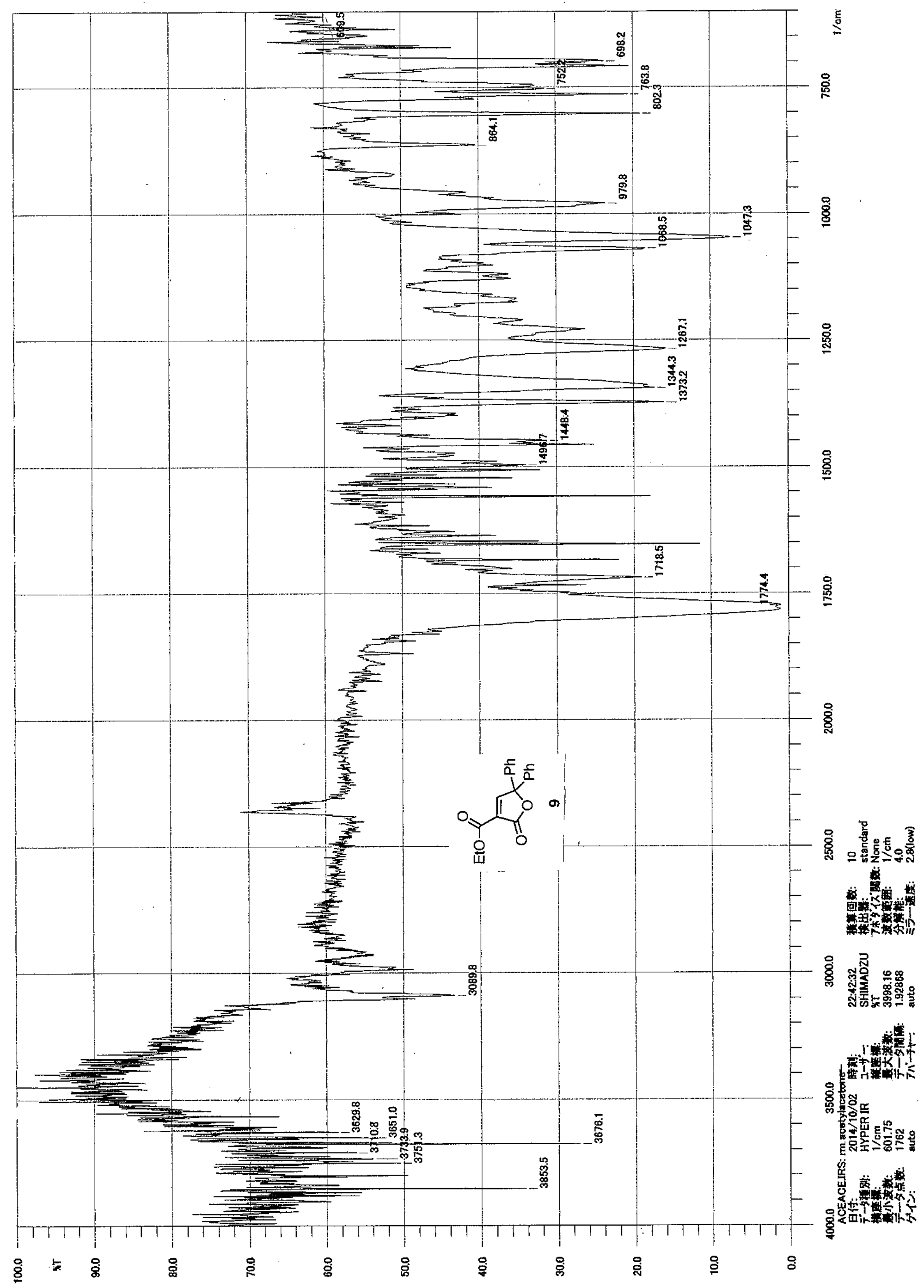




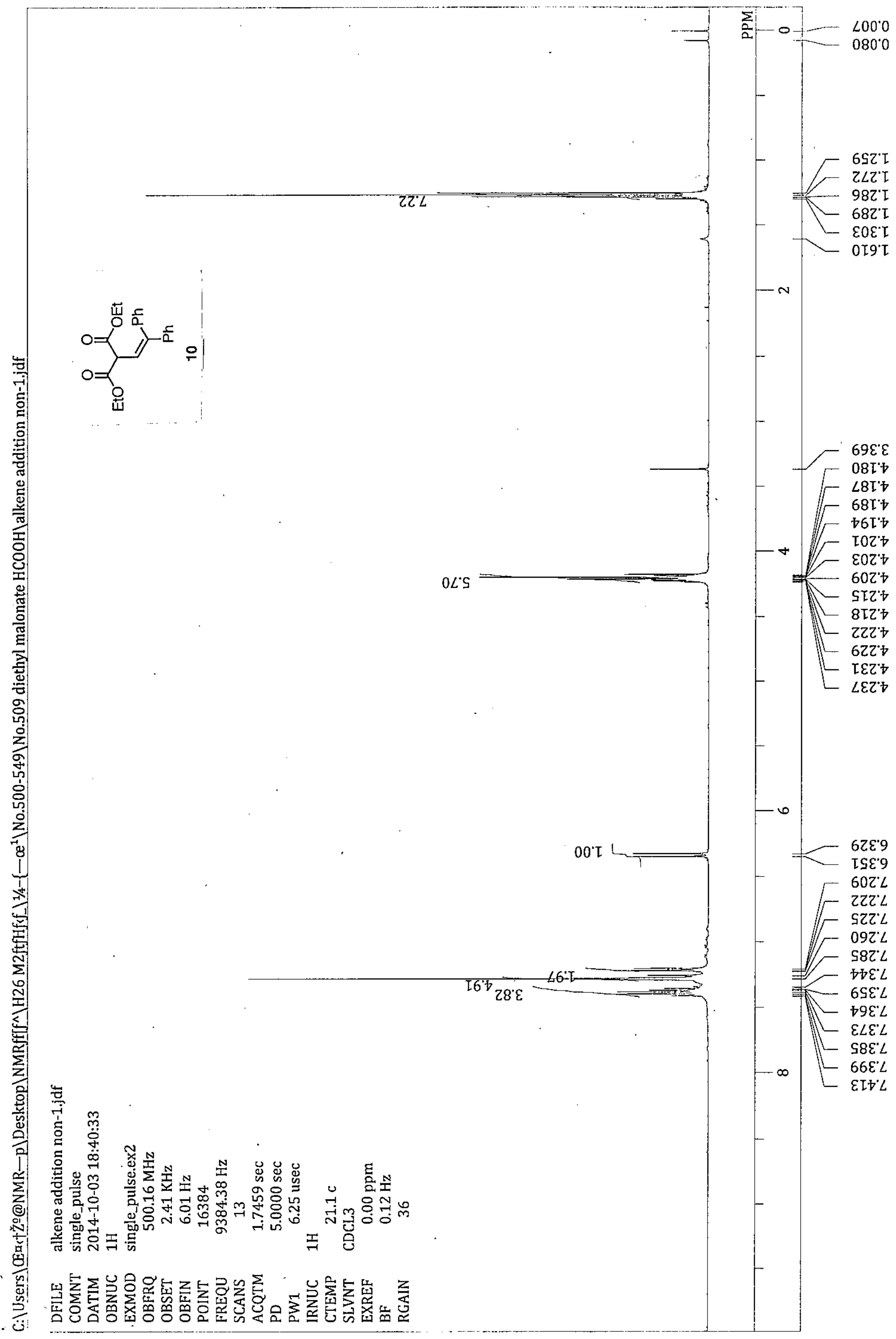




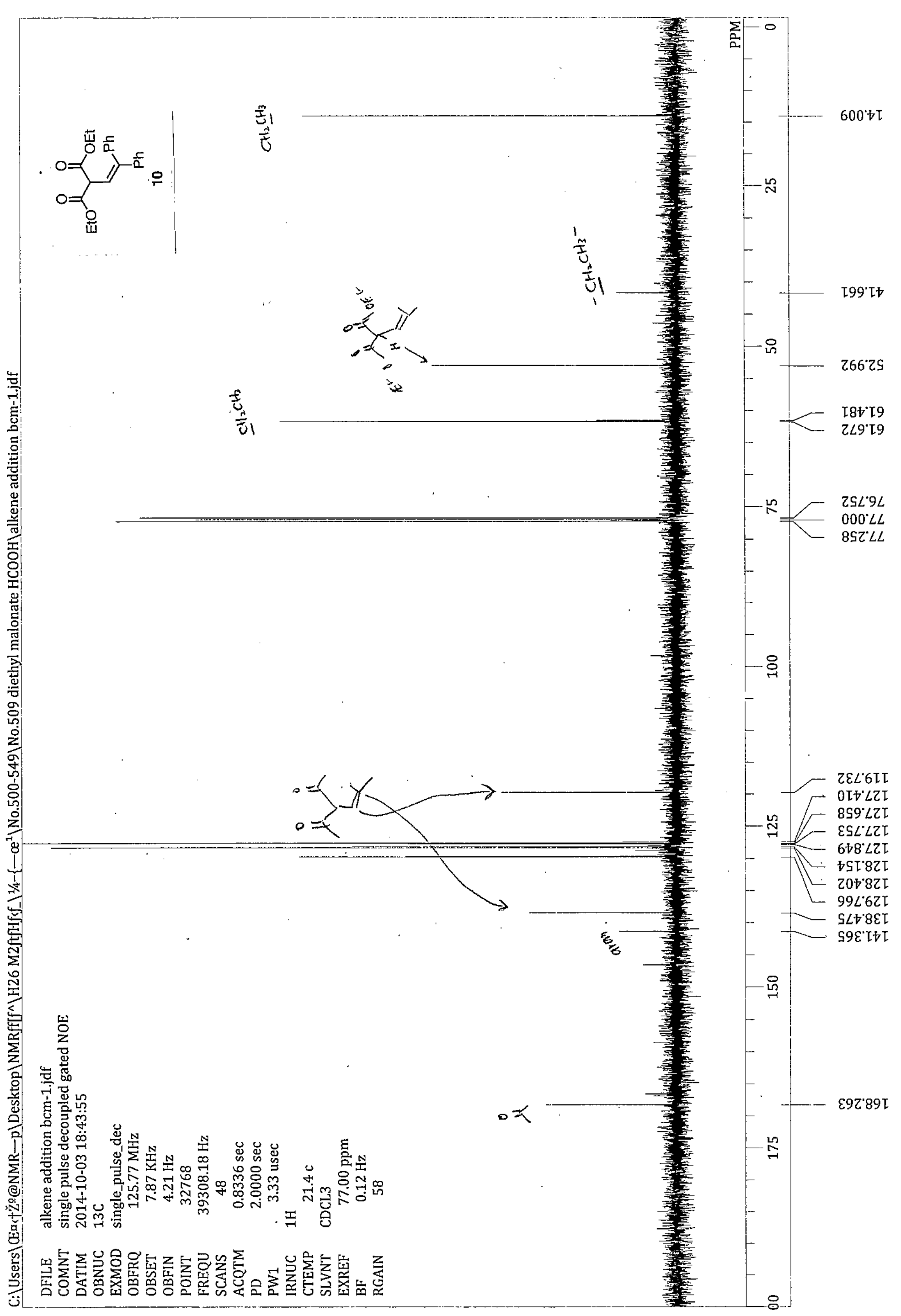




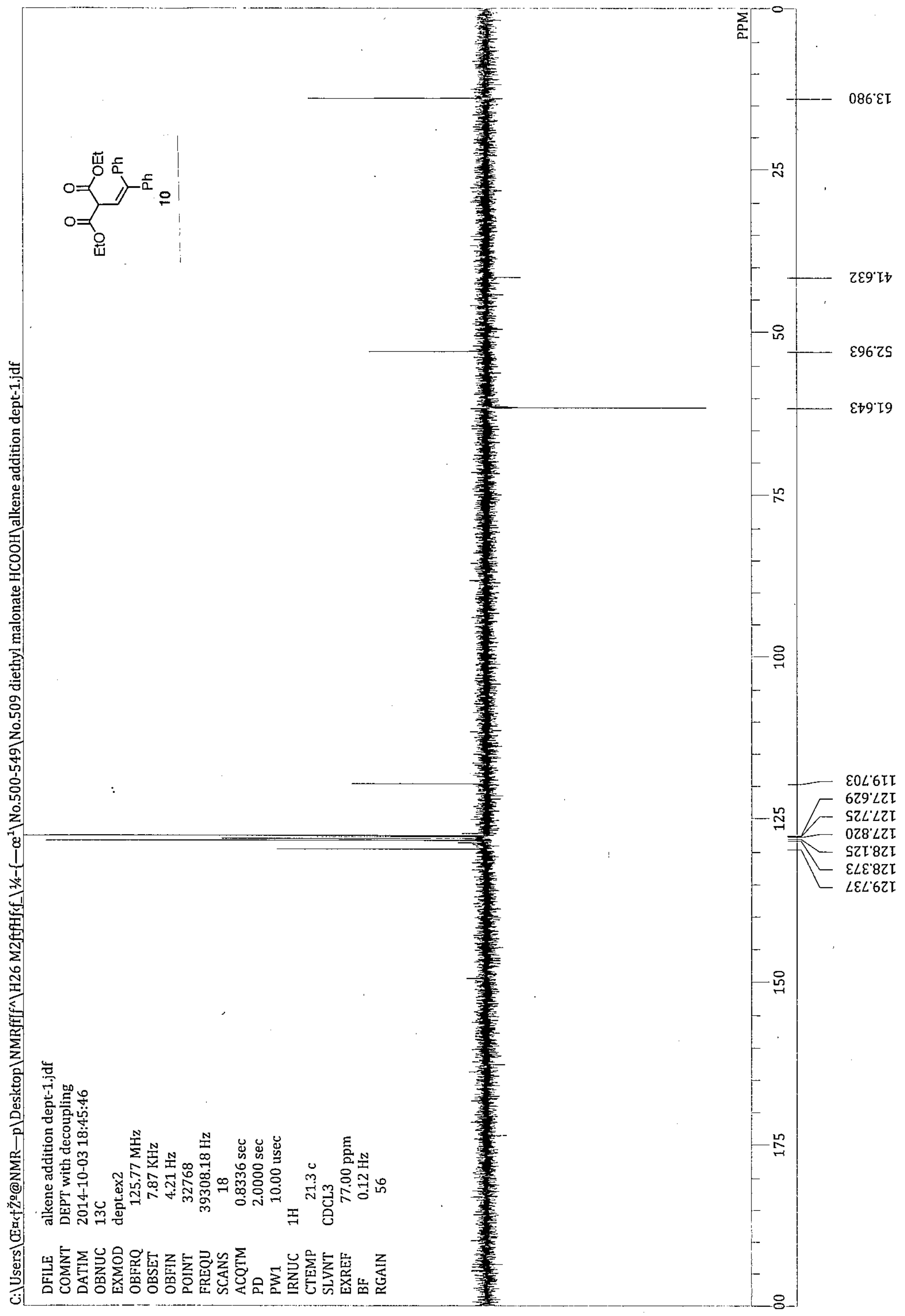

